\title{
A Multidimensional Identification Problem Related to a Hyperbolic Integro-Differential Equation
}

\author{
A. Lorenzi \\ Dedicated to $L$. von Wolfersdorf on the occasion of his retirement
}

\begin{abstract}
We prove a global in time existence and uniqueness theorem for the identification of a relaxation kernel $h$ entering a hyperbolic integro-differential equation, related to a convex cylinder with a smooth lateral surface, when the coefficient $h$ is assumed to depend on time and one space variable and general additional conditions are provided. A continuous dependence result for the identification problem is also stated. Finally, a separate proof concerning the existence and uniqueness of the solution to the related direct integro-differential problem is also given in a suitable functional space. Moreover, the dependence of such a solution with respect to the relaxation kernel is fully analysed.
\end{abstract}

Keywords: Linear integro-differential hyperbolic equations, determination of space- and timedependent relaxation kemels, global existence, uniqueness and continuous dependence results

AMS subject classiflcation: Primary $35 \mathrm{R} 30$, secondary $45 \mathrm{~K} 05,45 \mathrm{~N} 05$

\section{Introduction}

This paper is concerned with the identification of a unknown coefficient $h$ (the relaxation coefficient, depending on time and one space variable) appearing in the following integrodifferential equation related to the convex cylinder $\Omega=(0, \ell) \times \omega \subset \mathbb{R}^{n}(n \geq 2), \omega$ being an open bounded convex set in $\mathbb{R}^{n-1}$ of class $C^{1,1}$ :

$$
\begin{aligned}
D_{t}^{2} u(t, x, y)+A u(t, x, y) & +\int_{0}^{t} h(t-s, x) B u(s, x, y) d s \\
& +\int_{0}^{t} D_{x} h(t-s, x) C u(s, x, y) d s=f(t, x, y)
\end{aligned}
$$

for all $(t, x, y) \in[0, T] \times \Omega$. Here

A. Lorenzi: Università degli Studi, Dept. Math., via Saldini 50, 20133 Milano, Italia 


$$
\begin{aligned}
A= & -D_{x}\left[a_{1,1}(x) D_{x}\right] \\
& -\sum_{j=1}^{n-1} D_{x}\left[a_{1,1+j}(x, y) D_{y_{j}}\right]-\sum_{i=1}^{n-1} D_{y_{i}}\left[a_{1+i, 1}(x, y) D_{x}\right] \\
& -\sum_{i, j=1}^{n-1} D_{y_{i}}\left[a_{1+i, 1+j}(x, y) D_{y_{j}}\right]+a_{1}(x, y) D_{x} \\
& +\sum_{j=1}^{n-1} a_{1+j}(x, y) D_{y_{j}}+a_{0}(x, y) \\
B= & -D_{x}\left[b_{1,1}(x, y) D_{x}\right] \\
& -\sum_{j=1}^{n-1} D_{x}\left[b_{1,1+j}(x, y) D_{y_{j}}\right]-\sum_{i=1}^{n-1} D_{y_{i}}\left[b_{1+i, 1}(x, y) D_{x}\right] \\
& -\sum_{i, j=1}^{n-1} D_{y_{i}}\left[b_{1+i, 1+j}(x, y) D_{y_{j}}\right]+b_{1}(x, y) D_{x} \\
& +\sum_{j=1}^{n-1} b_{1+j}(x, y) D_{y_{j}}+b_{0}(x, y) \\
C= & c_{1}(x, y) D_{x}+\sum_{j=1}^{n-1} c_{1+j}(x, y) D_{y_{j}}+c_{0}(x, y)
\end{aligned}
$$

We note that $C$ is a linear (formal) first-order differential operator, while $A$ and $B$ are two linear (formal) second-order operators with principal parts in divergence form. We emphasize that the coefficient $a_{1,1}$ in $A$ depends on $x$ only, instead of on the pair $(x, y)$ as in the general case. The operator $A$ is uniformly elliptic, i.c.

$$
\alpha_{1}|\xi|^{2} \leq \sum_{i, j=1}^{n} a_{i, j}(x, y) \xi_{i} \xi_{j} \leq \alpha_{2}|\xi|^{2}
$$

for all $(x, y, \xi) \in(0, \ell) \times \omega \times \mathbb{R}^{n}$ where $\alpha_{1}>0$ and $\alpha_{2}>0$ with $\alpha_{1} \leq \alpha_{2}$ are two given constants.

We now prescribe the usual initial-boundary value conditions

$$
\begin{aligned}
u(0, x, y) & =u_{0}(x, y) & & ((x, y) \in \Omega) \\
D_{t} u(0, x, y) & =u_{1}(x, y) & & ((x, y) \in \Omega) \\
\frac{\partial u}{\partial \nu_{A}}(t, x, y) & =\frac{\partial u_{2}}{\partial \nu_{A}}(t, x, y) & & ((t, x, y) \in[0, T] \times \partial \Omega)
\end{aligned}
$$

where $u_{0}, u_{1}: \bar{\Omega} \rightarrow \mathbb{R}$ and $u_{2}:[0, T] \times \bar{\Omega} \rightarrow \mathbb{R}$ are given (smooth) functions and $\nu_{A}$ denotes the conormal vector associated with $A$ and $\Omega$. 
To determine the relaxation coefficient $h$ we assume that the two pieces of additional information

$$
\begin{aligned}
\Phi[u(t, \cdot)](x) & =\varphi(t, x) & & ((t, x) \in[0, T] \times(0, \ell)) \\
\Psi[u(t, \cdot, \cdot)] & =\psi(t) & & (t \in[0, T])
\end{aligned}
$$

are available where $\Phi$ is a linear operator acting on the variable $y$ only, while $\Psi$ is a linear functional acting on all the space variables. Examples of admissible $\Phi$ and $\Psi$ are

$$
\begin{aligned}
\Phi[v](x) & =\int_{\omega} \lambda(x, y) v(x, y) d y \quad(x \in(0, \ell)) \\
\Psi[v] & =\int_{\Omega} \rho(x, y) v(x, y) d x d y
\end{aligned}
$$

where $\lambda: \omega \rightarrow \mathbb{R}$ and $\rho: \Omega \rightarrow \mathbb{R}$ denote two (smooth) assigned functions.

We observe that our choice not to explicitly define operator $\Phi$ and functional $\Psi$ allows a large variety of applications, the actual choice of $\Phi$ and $\Psi$ being left to the user. Then we observe that our data have to fulfil the consistency conditions

$$
\begin{aligned}
\frac{\partial u_{j}}{\partial \nu_{A}}(x, y) & =D_{\imath}^{j} \frac{\partial u_{2}}{\partial \nu_{A}}(0, x, y) & & ((x, y) \in \partial \Omega) \\
D_{\imath}^{j} \varphi(0, x) & =\Phi\left[u_{j}\right](x) & & (x \in[0, \ell]) \\
D_{\imath}^{j} \psi(0) & =\Psi\left[u_{j}\right] & &
\end{aligned}
$$

for $j=1,2$.

Remark 1.1. Further consistency conditions may occur in some specific cases (cf. Section 2/Remark 2.1).

Finally, we observe that the determination of the relaxation kernel $h$ (depending on time and one space variable, the axial one) is of interest in applied problems related to stratified media. In this case the operators $A, B$ and $C$ often take the particular forms

$$
\begin{aligned}
& A=-D_{y_{0}}\left(\tilde{a}_{1,1}\left(y_{0}\right) D_{y_{0}}\right)-\sum_{i, j=1}^{n-1} D_{y_{i}}\left(\tilde{a}_{i, j}\left(y_{0}\right) D_{y_{j}}\right) \\
& B=-\sum_{i, j=0}^{n-1} D_{y_{i}}\left(\tilde{b}_{i, j}\left(y_{0}\right) D_{y_{j}}\right) \\
& C=-\sum_{i=0}^{n-1} \tilde{c}_{j}\left(y_{0}\right) D_{y_{j}}
\end{aligned}
$$

where $y_{0}=x$ and $D_{y_{0}}=D_{x}$.

The class of problems dealt with in this paper seems, at present, not to have been widely investigated, in contrast to the case where the relaxation kernel depends on time, 
only (cf., e.g., $[4-6,8,9,14-16])$. Therefore this paper wants to contribute to develop the studies in this area. We observe that some related papers are $[2,11,12]$. In [2] and [11] the authors deal with the problem of the diffusion of electromagnetic waves in dispersive media and are concerned with the determination of a relaxation kernel depending on time and one space variable. The same identification problem is treated in [12], but relatively to stratified viscoelastic materials and specific assumptions on the data.

We conclude this section by noting that Theorems 2.1 and 2.2 in Section 2 report the main results involving the identification problem. They are concerned with a global existence and uniqueness result as well as a global continuous dependence result for the solution $(u, h)$ to the identification problem (1.1), (1.6) - (1.10). Furthermore, some applications, related to the specific operator $\Phi$ and functional $\Psi$ defined by (1.11) and (1.12), are reported in Section 6.

Finally, the well-posedness of the direct problem (1.1), (1.6) - (1.8) is ensured by Theorem 4.2 in Section 4, which provides also the continuous dependence of the solution $u$ on the relaxation kernel $h$.

\section{The main result}

We state in this section our global in time existence and uniqueness results related to problem (1.1), (1.6) - (1.10). For this purpose we assume that the coefficients of our operators $A, B$ and $C$ enjoy, in addition to (1.5), also the properties

$$
\begin{array}{ll}
a_{1,1} \in C^{1,1}([0, \ell]), a_{i, j}, b_{i, j} \in C^{0,1}(\bar{\Omega}), a_{i, j}=a_{j, i} & (i, j=2, \ldots, n) \\
D_{x} a_{1, j+1} \in C^{0,1}(\bar{\Omega}), D_{x} D_{y_{i}} a_{1, j+1} \in L^{\infty}(\Omega) & (j=1, \ldots, n-1) \\
a_{j}, b_{j}, c_{j} \in L^{\infty}(\Omega) & (j=0, \ldots, n) .
\end{array}
$$

Here $C^{m, 1}(\overline{\mathcal{O}}), \mathcal{O}$ being an open bounded set in $\mathbb{R}^{d}$, denotes the subspace of all functions in $C^{m}(\mathcal{O})$ whose derivatives of order $m$ can be extended to $\overline{\mathcal{O}}$ by Lipschitz continuous functions.

We now recall that the open set $\omega$ is convex and assume that the operator $\Phi$ and the functional $\Psi$ enjoy the properties

$$
\begin{aligned}
& \Phi \in \bigcap_{j=0}^{2} \mathcal{L}\left(H^{j}(\Omega) ; H^{j}(0, \ell)\right) \text { and } \Psi \in L^{2}(\Omega)^{*} \\
& \Phi[h u]=h \Phi[u] \quad \text { for all }(h, u) \in L^{2}(0, \ell) \times L^{2}(\Omega) \\
& \Phi[u](k \ell)=\Phi[u(k \ell, \cdot)](k \ell) \text { for all } u \in L^{2}\left(\omega, H^{1}(0, \ell)\right) \\
& D_{x} \Phi[u](k \ell)=\Phi\left[D_{x} u\right](k \ell)+\Phi_{0, k}[u(k \ell, \cdot)], \quad \Phi_{0, k} \in L^{2}(\omega)^{*} \\
& \quad \text { for all } u \in L^{2}\left(\omega, H^{1}(0, \ell)\right) \text { and } k=0,1 \\
& \Phi A=A_{1} \Phi+\Phi_{1} \frac{\partial}{\partial \nu_{A}}+\Phi_{2} \text { on } H^{2}(\Omega)
\end{aligned}
$$




$$
\begin{aligned}
& \Phi_{1} \in \mathcal{L}\left(H^{\frac{1}{2}}((0, \ell) \times \partial \omega) ; L^{2}(0, \ell)\right), \quad \Phi_{2} \in \mathcal{L}\left(H^{1}(\Omega) ; L^{2}(0, \ell)\right) \\
& \Psi A=\Psi_{1} \frac{\partial}{\partial \nu_{A}}+\Psi_{2} \text { on } H^{2}(\Omega), \quad \Psi_{1} \in H^{\frac{1}{2}}(\partial \Omega)^{*}, \quad \Psi_{2} \in H^{1}(\Omega)^{*}
\end{aligned}
$$

where

$$
A_{1}=-D_{x}\left[a_{1,1}(x) D_{x}\right]
$$

Moreover, $\mathcal{L}(X ; Y), X^{*}$ and $H^{m}(\mathcal{O})$ denote the Banach space of all linear bounded operators between the Banach spaces $X$ and $Y$, the dual space to $X$ and the Sobolev space of order $m$ related to $L^{2}(\mathcal{O})$, respectively.

Assume now that the data $f, u_{0}, u_{1}, u_{2}, \varphi, \psi$ related to the identification problem (1.1), (1.6) - (1.10), enjoy the properties

$$
\begin{aligned}
& f \in W^{2,1}\left((0, T) ; L^{2}(\Omega)\right), \quad u_{0} \in H^{2}(\Omega), \quad u_{1} \in H^{2}(\Omega), \quad u_{2} \in \mathcal{U}^{4,1}(T, \Omega) \\
& A u_{0}-f(0, \cdot, \cdot) \in H^{1}(\Omega), \quad D_{t}\left(D_{t}^{2} u_{2}+A_{0} u_{2}\right) \in W^{2,1}\left((0, T) ; L^{2}(\Omega)\right) \\
& \varphi \in \mathcal{U}^{4,1}(T,(0, \ell)), \quad \psi \in W^{4,1}(0, T)
\end{aligned}
$$

where $A_{0}$ denotes the principal part of the operator $A$ (cf. (1.2)) and the Banach spaces $\mathcal{U}^{s, p}(T, \mathcal{O}), \mathcal{O}$ being an open set in $\mathbb{R}^{d}$, are defined for any $s \in \mathbb{N} \cap[2,+\infty)$ by

$$
\begin{aligned}
& \mathcal{U}^{s, p}(T, \mathcal{O})= \\
& \left\{\begin{array}{l}
W^{s, p}\left((0, T) ; L^{2}(\mathcal{O})\right) \cap W^{s-1, p}\left((0, T) ; H^{1}(\mathcal{O})\right) \cap W^{s-2, p}\left((0, T) ; H^{2}(\mathcal{O})\right) \\
C^{s}\left([0, T] ; L^{2}(\mathcal{O})\right) \cap C^{s-1}\left([0, T] ; H^{1}(\mathcal{O})\right) \cap C^{s-2}\left([0, T] ; H^{2}(\mathcal{O})\right)
\end{array}\right.
\end{aligned}
$$

according as $p \in[1,+\infty)$ or $p=+\infty$.

Remark 2.1. From assumptions (2.6), (2.7) we easily derive the chain of equalities

$$
\begin{aligned}
\Phi\left[\frac{\partial u}{\partial \nu_{A}}(t, \cdot, \cdot)\right](k \ell)= & \Phi\left[\frac{\partial u}{\partial \nu_{A}}(t, k \ell, \cdot)\right](k \ell) \\
= & a_{1,1}(k \ell) \Phi\left[D_{x} u(t, k \ell, \cdot)\right](k \ell) \\
& +\Phi\left[\sum_{j=1}^{n-1} a_{1,1+j}(k \ell, \cdot) D_{y_{j}} u(t, k \ell, \cdot)\right](k \ell) \\
= & a_{1,1}(k \ell) D_{x} \Phi[u(t, \cdot, \cdot)](k \ell)-a_{1,1}(k \ell) \Phi_{0}[u(t, \cdot, \cdot)](k \ell) \\
& +\Phi\left[\sum_{j=1}^{n-1} a_{1,1+j}(k \ell, \cdot) D_{y_{j}} u(t, \cdot)\right](k \ell)
\end{aligned}
$$

for $t \in[0, T]$ and $k=0,1$. Observe that, if the vector valued function $\left(a_{1,2}, \ldots, a_{1, n}\right)$ vanishes everywhere on $\{0, \ell\} \times \omega$ and $\Phi$ commutes with $D_{x}$ at $x=k \ell(k=0,1)$, then from (2.16) we easily deduce the consistency condition

$$
\left.\begin{array}{l}
\Phi\left[\frac{\partial u_{2}}{\partial \nu_{A}}(t, \cdot, \cdot)\right](k \ell)=a_{1,1}(k \ell) D_{x} \varphi(t, k \ell) \quad(t \in[0, T] ; k=0,1) \\
\text { if } a_{1, j}=0 \text { on }\{0, \ell\} \times \omega(j=2, \ldots, n) \text { and } \Phi \text { commutes with } D_{x} \text { at } x=k \ell .
\end{array}\right\}
$$


On the contrary, if $\left(a_{1,2}, \ldots, a_{1, n}\right)$ does not coincide with the null function on $\{0, \ell\} \times \omega$ or $\Phi$ does not commute with $D_{x}$ at $x=k \ell(k=0,1)$, then condition (2.16) prescribes the values of two (known) functionals, related to $\Phi, \Phi_{0}$ and the unknown $u$, on the bases of the cylinder $\Omega$.

Finally, assume that $u_{0}$ satisfies the conditions

$$
\begin{aligned}
m_{1}\left(u_{0}\right)(x) & :=\left|\Phi\left[C u_{0}\right](x)\right| \geq \bar{m}_{1}>0 \quad(x \in[0, \ell]) \\
m_{2}\left(u_{0}\right) & :=\Psi\left[J\left(u_{0}\right)\right] \neq 0
\end{aligned}
$$

for some constant $\bar{m}_{1}>0$ where

$$
J\left(u_{0}\right)(x, y)=\left(B u_{0}(x, y)-\frac{\Phi\left[B u_{0}\right](x)}{\Phi\left[C u_{0}\right](x)} C u_{0}(x, y)\right) \exp \left(-\int_{0}^{x} \frac{\Phi\left[B u_{0}\right](\xi)}{\Phi\left[C u_{0}\right](\xi)} d \xi\right) .
$$

Remark 2.2. According to properties (2.3) - (2.4) we easily get the equations

$$
\Phi\left[J\left(u_{0}\right)\right](x)=\exp \left(-\int_{0}^{x} \frac{\Phi\left[B u_{0}\right](\xi)}{\Phi\left[C u_{0}\right](\xi)} d \xi\right) \Phi\left(B u_{0}-\frac{\Phi\left[B u_{0}\right]}{\Phi\left[C u_{0}\right]} C u_{0}\right)(x)=0
$$

for $x \in(0, \ell)$. Consequently, in order that assumption (2.19) may be satisfied, the functional $\Psi$ must be linearly functionally independent of the operator $\Phi$ in the sense that there exists no functional $\Lambda \in L^{2}(0, \ell)^{*}$ with $\Psi=\Lambda \Phi$. Otherwise, we should get $\Psi\left[J\left(u_{0}\right)\right]=0$. In particular, when $\Phi$ and $\Psi$ admit the integral representations (1.11) and (1.12), this means that no function $\rho$ of the form $\rho(x, y)=\rho_{1}(x) \lambda(x, y)$ is allowed.

Remark 2.3. When $\Phi$ admits the representation (1.11), a necessary condition for assumption (2.18) to be satisfied is that there exists no $x_{0} \in[0, \ell]$ such that $\lambda\left(x_{0}, y\right)=0$ for any $y \in \bar{\omega}$.

We can now state the two main results of this paper involving our identification problem: a global existence and uniqueness theorem and the corresponding continuous dependence result.

Theorem 2.1. Let assumptions (1.5) and (2.1)-(2.10) be fulfilled. Assume further that the data enjoy properties (2.12) - (2.14), fulfil the consistency conditions (1.13)(1.15), (2.17) and the inequalities (2.18) and (2.19). Then problem (1.1), (1.6) - (1.10) admits a unique solution $(u, h) \in \mathcal{U}^{3, \infty}(T, \Omega) \times W^{1,1}\left((0, T) ; H^{1}(0, \ell)\right)$.

Theorem 2.2. Let $\left(f_{j}, u_{0, j}, u_{1, j}, u_{2, j}, \varphi_{j}, \psi_{j}\right)(j=1,2)$ be two sixtuplets satisfying properties (2.12) - (2.14), (2.18), (2.19) with

$$
0<\bar{m}_{1} \leq \inf _{x \in[0, \ell]}\left[m_{1}\left(u_{0,1}\right)(x), m_{1}\left(u_{0,2}\right)(x)\right]
$$

as well as consistency conditions $(1.13)-(1.15),(2.17)$. Then the solutions $\left(u_{j}, h_{j}\right)$ corresponding to the data $\left(f_{j}, u_{0, j}, u_{1, j}, u_{2, j}, \varphi_{j}, \psi_{j}\right)(j=1,2)$ satisfy the estimate

$$
\begin{aligned}
\| u_{2}- & u_{1}\left\|_{u^{3, \infty}(T, \Omega)}+\right\| h_{2}-h_{1} \|_{W^{1,1}\left((0, T) ; H^{\prime}(0, \ell)\right)} \\
\leq & C\left(T, \bar{m}_{1}, \bar{m}_{2}\right) \\
\quad & \times\|\|\left(f_{2}-f_{1}, u_{0,2}-u_{0,1}, u_{1,2}-u_{1,1}, u_{2,2}-u_{2,1}, \varphi_{2}-\varphi_{1}, \psi_{2}-\psi_{1}\right) \|
\end{aligned}
$$


where

$$
0<\bar{m}_{2} \leq \min \left(\left|m_{2}\left(u_{0,1}\right)\right|,\left|m_{2}\left(u_{0,2}\right)\right|\right)
$$

and

$$
\begin{aligned}
\left\|\left(f, u_{0}, u_{1}, u_{2}, \varphi, \psi\right)\right\| \leq & \|f\|_{W^{2,1}\left((0, T) ; L^{2}(\Omega)\right)}+\sum_{j=1}^{2}\left\|u_{j}\right\|_{H^{2}(\Omega)} \\
& +\left\|u_{2}\right\|_{u^{4,1}(T, \Omega)}+\left\|A u_{2}-f(0, \cdot)\right\|_{H^{1}(\Omega)} \\
& +\left\|D_{t}\left(D_{t}^{2} u_{2}+A u_{2}\right)\right\|_{W^{2,1}\left((0, T) ; L^{2}(\Omega)\right)} \\
& +\|\varphi\|_{u^{4,1}(T,(0, \ell))}+\|\psi\|_{W^{4,1}(0, T)}
\end{aligned}
$$

Remark 2.4. To prove our existence and uniqueness result it is essential that condition (2.18) should be satisfied. This explains why we have chosen a particular non-smooth domain - a cylinder - instead of a general regular one. Indeed, assume for a moment that our domain were smooth and of the form $\Omega=\bigcup_{x \in[0, \ell]} \omega(x)$ with $m_{n-1}(\omega(x)) \rightarrow 0 x \rightarrow 0$ or $x \rightarrow \ell, m_{n-1}$ denoting the $(n-1)$-dimensional Lebesgue measure.

We could replace condition (1.11) in a natural way with the following

$$
\Phi[v](x)=\int_{\omega(x)} \lambda(x, y) v(x, y) d y \quad(x \in(0, \ell))
$$

In this case, for regular kernels $\lambda, \Phi[v]$ would not be bounded away from 0 for any function $v$. As a consequence, if we would want to deal with smooth domains, they should be of barrel type, only. Morcover, such a choice would cause a remarkable complication in the treatment of our identification problem (cf. assumption (2.7)).

Remark 2.5. Note that estimate (2.20) takes into account all the metric spaces involved in conditions (2.12)-(2.14). Moreover, if $\bar{m}_{1}$ and $\bar{m}_{2}$ are lower uniform bounds for $m_{1}\left(u_{0}\right)$ and $m_{2}\left(u_{0}\right)$ as $u_{0}$ runs on the set of all admissible $u_{0},(2.20)$ ensures that the mapping data $\rightarrow$ solution is Lipschitz continuous when the set of data is provided with the metrics induced by the norm in (2.21).

To conclude this section we observe that, because of its length, we are forced to give only a very short outline of the proof of Theorem 2.2 (cf. the end of section 6). However, we note that Theorem 2.2 could be proved following the same ideas as in the proof of the existence and uniqueness Theorem 2.1. About the proof of the latter we want to give here a short outline as well as some comments.

i) Section 3 will be devoted to transforming our original problem into an equivalent one for a triplet $\left(v, k_{0}, k_{1}\right)$, where $v=D_{t} u$. It will consist of an integro-differential equation for $v$ as well as of a fixed-point system for $\left(k_{0}, k_{1}\right)$. This process will explain also the necessity of the additional condition (1.12), which, at a first sight, might seem superfluous.

ii) In Section 4 we shall solve the (direct) integro-differential problem for $v$ in a functional space ensuring the maximal spatial regularity allowed in a non-smooth situation as ours is. For this purpose we recall that, according to the results in [7: Theorem 3.2.1.3], the maximal spatial regularity for $u$, in a (general) convex cylinder, 
is expressed by the membership in $H^{2}(\Omega)$. Moreover, some basic weighted estimates will be proved. In particular they will establish the continuous dependence of $v$ on the relaxation kernel $h$.

iii) In Section 5 we will transform the problem for $\left(v, k_{0}, k_{1}\right)$ in a system of operator fixed-point equations for the pair $\left(k_{0}, k_{1}\right)$. Such a system will be solved (in suitable weighted spaces) using the Banach's Contraction Principle.

iv) Section 6 will be devoted to determining suitable conditions on the kernels $\lambda$ and $\rho$ in order the explicit operator $\Phi$ and $\Psi$ defined by (1.11) and (1.12) may satisfy the basic conditions (2.4) - (2.10).

\section{An equivalence result}

Assume that $(u, h) \in \mathcal{U}^{3, \infty}(T, \Omega) \times W^{1,1}\left((0, T) ; H^{1}(0, \ell)\right)(\mathrm{cf}$. (2.15)) is a solution to the identification problem (1.1), (1.6) - (1.10) and introduce the new unknown function

$$
v(t, x, y)=D_{t} u(t, x, y) \quad \Longleftrightarrow u(t, x, y)=u_{0}(x, y)+\int_{0}^{t} v(s, x, y) d s
$$

Then the pair $(v, h) \in \mathcal{U}^{2, \infty}(T, \Omega) \times W^{1,1}\left((0, T) ; H^{1}(0, \ell)\right)$ solves the following system of equations:

$$
\begin{gathered}
D_{t}^{2} v(t, x, y)+A v(t, x, y) \\
+\int_{0}^{t} h(t-s, x) B v(s, x, y) d s \\
+\int_{0}^{t} D_{x} h(t-s, x) C v(s, x, y) d s \\
+D_{x} h(t, x) C u_{0}(x, y)+h(t, x) B u_{0}(x, y)=D_{t} f(t, x, y)
\end{gathered}
$$

for $(t, x, y) \in[0, T] \times \Omega)$

$$
\begin{aligned}
& v(0, x, y)=u_{1}(x, y) \quad((x, y) \in \Omega) \\
& D_{t} v(0, x, y)=-A u_{0}(x, y)+f(0, x, y) \quad((x, y) \in \Omega) \\
& \frac{\partial v}{\partial \nu_{A}}(t, x, y)=D_{t} \frac{\partial u_{2}}{\partial \nu_{A}}(t, x, y) \quad((t, x, y) \in[0, T] \times \partial \Omega) \\
& D_{x} h(t, x) \Phi\left[C u_{0}\right](x)+h(t, x) \Phi\left[B u_{0}\right](x) \\
& =-D_{t}^{3} \varphi(t, x)-D_{t} A_{1} \varphi(t, x)-\Phi_{1}\left[D_{t} \frac{\partial u_{2}}{\partial \nu_{A}}(t, \cdot)\right](x) \\
& \quad-\Phi_{2}[v(t, \cdot)](x)-\int_{0}^{t} h(t-s, x) \Phi[B v(s, \cdot)](x) d s \\
& \quad-\int_{0}^{t} D_{x} h(t-s, x) \Phi[C v(s, \cdot)](x) d s+\Phi\left[D_{t} f(t, \cdot)\right](x) \\
& :=N_{1}(v, h)(t, x)
\end{aligned}
$$


for $(t, x) \in[0, T] \times(0, \ell)$

$$
\begin{aligned}
\Psi[ & \left.D_{x} h(t, \cdot) C u_{0}\right]+\Psi\left[h(t, \cdot) B u_{0}\right] \\
= & -D_{t}^{3} \psi(t)-\Psi_{1}\left[D_{t} \frac{\partial u_{2}}{\partial \nu_{A}}(t, \cdot, \cdot)\right] \\
& -\Psi_{2}[v(t, \cdot, \cdot)]-\Psi\left[\int_{0}^{t} h(t-s, \cdot) B v(s, \cdot, \cdot) d s\right] \\
& -\Psi\left[\int_{0}^{t} D_{x} h(t-s, \cdot) C v(s, \cdot, \cdot) d s\right]+\Psi\left[D_{t} f(t, \cdot, \cdot)\right] \\
& :=N_{2}(v, h)(t)
\end{aligned}
$$

for $t \in[0, T]$. We note that equations (3.6) and (3.7) can be derived applying the operator $\Phi$ and the functional $\Psi$ to both members in (3.2) and using assumptions (2.4) - (2.10).

Assume now that $(v, h) \in \mathcal{U}^{2, \infty}(T, \Omega) \times W^{1,1}\left((0, T) ; H^{1}(0, \ell)\right)$ is a solution to problem (3.2) - (3.7) and introduce the function $u \in \mathcal{U}^{3, \infty}(T, \Omega)$ defined in (3.1). Owing to consistency conditions (1.13), from (3.2) - (3.4) we immediately deduce that $u$ solves equations (1.1), (1.6) - (1.8). We integrate, then, with respect to $t$ equation (3.6) (where we have set $v=D_{t} u$ ) and use identity (2.8) and consistency conditions (1.13) - (1.15). We get the equation

$$
\begin{aligned}
D_{t}^{2} \varphi(t, x)+\Phi[A u(t, \cdot)](x) & +\int_{0}^{t} h(t-s, x) \Phi[B u(s, \cdot)](x) d s \\
& +\int_{0}^{t} D_{x} h(t-s, x) \Phi[C u(s, \cdot)](x) d s=\Phi[f(t, \cdot)](x)
\end{aligned}
$$

for $(t, x) \in[0, T] \times(0, \ell)$. Apply now operator $\Phi$ to both members in (1.1) and subtract memberwise (3.8) from the equation just found. We easily get the equation

$$
D_{t}^{2} \Phi[u(t, \cdot)](x)=D_{t}^{2} \varphi(t, x)
$$

for $(t, x) \in[0, T] \times(0, \ell)$. Using conditions (1.6), (1.7) and (1.14), we deduce that $u$ satisfies the additional information (1.9). Performing similar computations, we can show that $u$ satisfies also equation (1.10). Summing up, we have shown that the identification problems (1.1), (1.6) - (1.10) and (3.2) - (3.7) are equivalent.

Setting $t=0$ in equations (3.6) and (3.7) we get the following (operator) differential system for $h(0, \cdot)$ :

$$
\begin{aligned}
& D_{x} h(0, x) \Phi\left[C u_{0}\right](x)+h(0, x) \Phi\left[B u_{0}\right](x) \\
&=-D_{t}^{3} \varphi(0, x)-D_{t} A_{1} \varphi(0, x) \\
& \quad-\Phi_{1}\left[D_{t} \frac{\partial u_{2}}{\partial \nu_{A}}(t, \cdot)\right](x)-\Phi_{2}\left[u_{1}\right](x)+\Phi\left[D_{t} f(0, \cdot)\right](x) \\
&:= \ell_{1}(x) \\
& \Psi\left[D_{x} h(0, \cdot) C u_{0}+h(0, \cdot) B u_{0}\right] \\
&=-D_{t}^{3} \psi(0)-\Psi_{1}\left[D_{t} \frac{\partial u_{2}}{\partial \nu_{A}}(0, \cdot, \cdot)\right]-\Psi_{2}\left[u_{1}\right]+\Psi\left[D_{t} f(0, \cdot, \cdot)\right]
\end{aligned}
$$


Integrating the differential equation in (3.10), we obtain the following general integral depending on an arbitrary constant $c$ (cf. assumption (2.18)):

$$
\begin{aligned}
h(0, x)= & c \exp \left(-\int_{0}^{x} \frac{\Phi\left[B u_{0}\right](\xi)}{\Phi\left[C u_{0}\right](\xi)} d \xi\right) \\
& +\int_{0}^{x} \exp \left(-\int_{\eta}^{x} \frac{\Phi\left[B u_{0}\right](\xi)}{\Phi\left[C u_{0}\right](\xi)} d \xi\right) \frac{\ell_{1}(\eta)}{\Phi\left[C u_{0}\right](\xi)} d \eta
\end{aligned}
$$

where

$$
\begin{aligned}
\ell_{1}(x)= & -D_{\imath}^{3} \varphi(0, x)-D_{t} A_{1} \varphi(0, x)-\Phi_{1}\left[D_{t} \frac{\partial u_{2}}{\partial \nu_{A}}(0, \cdot)\right](x) \\
& -\Phi_{2}\left[u_{1}\right](x)+\Phi\left[D_{t} f(0, \cdot)\right](x)
\end{aligned}
$$

for $x \in[0, \ell]$. Substituting this representation of $h(0, \cdot)$ into $(3.11)$ and using condition (2.19), we can easily compute $c$ as

$$
\begin{aligned}
c= & {\left[m_{2}\left(u_{0}\right)\right]^{-1} } \\
& \times\left\{-\Psi\left[\ell_{2}\right]-D_{t}^{3} \psi(0)-\Psi_{1}\left[D_{t} \frac{\partial u_{2}}{\partial \nu_{A}}(0, \cdot, \cdot)\right]-\Psi_{2}\left[u_{1}\right]+\Psi\left[D_{t} f(0, \cdot, \cdot)\right]\right\}
\end{aligned}
$$

where

$$
\begin{aligned}
\ell_{2}(x, y)= & \frac{C u_{0}(x, y)}{\Phi\left[C u_{0}\right](x)} \ell_{1}(x)+\left(B u_{0}(x, y)-\frac{\Phi\left[B u_{0}\right](x)}{\Phi\left[C u_{0}\right](x)} C u_{0}(x, y)\right) \\
& \times \int_{0}^{x} \exp \left(-\int_{\eta}^{x} \frac{\Phi\left[B u_{0}\right](\xi)}{\Phi\left[C u_{0}\right](\xi)} d \xi\right) \frac{\ell_{1}(\eta)}{\Phi\left[C u_{0}\right](\eta)} d \eta
\end{aligned}
$$

for $(x, y) \in \Omega)$. Hence, from (3.12) and (3.14) we derive the initial value for $h(0, \cdot)$

$$
\begin{aligned}
h(0, x)= & {\left[m_{1}\left(u_{0}\right)\right]^{-1}\left\{-\Psi\left[\ell_{2}\right]-D_{t}^{3} \psi(0)-\Psi_{1}\left[D_{t} \frac{\partial u_{2}}{\partial \nu_{A}}(0, \cdot, \cdot)\right]\right.} \\
& \left.-\Psi_{2}\left[u_{1}\right]+\Psi\left[D_{t} f(0 ; \cdot, \cdot)\right]\right\} \exp \left(-\int_{0}^{x} \frac{\Phi\left[B u_{0}\right](\xi)}{\Phi\left[C u_{0}\right](\xi)} d \xi\right) \\
& +\int_{0}^{x} \exp \left(-\int_{\eta}^{x} \frac{\Phi\left[B u_{0}\right](\xi)}{\Phi\left[C u_{0}\right](\xi)} d \xi\right) \frac{\ell_{1}(\eta)}{\Phi\left[C u_{0}\right](\eta)} d \eta \\
= & : h_{0}(x)
\end{aligned}
$$

Introduce then the new unknowns

$$
\left.\begin{array}{rl}
k_{0}(t) & =h(t, 0) \\
k_{1}(t, x) & =D_{x} h(t, x)
\end{array}\right\} \Longleftrightarrow h(t, x)=k_{0}(t)+\int_{0}^{x} k_{1}(t, \xi) d \xi:=H(k)(t, x)
$$

for $(t, x) \in[0, T] \times(0, \ell)$. 
Remark 3.1. Since $h(0, \cdot)=h_{0}$, we immediately derive the initial conditions

$$
\left.\begin{array}{r}
k_{0}(0)=h_{0}(0) \\
k_{1}(0, \cdot)=D_{x} h_{0}
\end{array}\right\}
$$

These conditions, in turn, implies the equation $H(k)(0, \cdot)=h_{0}$.

We use now definitions (3.17) and solve system (3.6) - (3.7) for $k=\left(k_{0}, k_{1}\right)$ in terms of the right-hand sides $\left(N_{1}(v, H(k)), N_{2}(v, H(k))\right)$. Explicitly, we get the system

$$
\begin{aligned}
& \ddot{k}_{1}(t, x) \Phi\left[C u_{0}\right](x)+\Phi\left[B u_{0}\right](x) 1 * k_{1}(t, x) \\
& =-k_{0}(t) \Phi\left[B u_{0}\right](x)+N_{1}(v, H(k))(t, x) \\
& \Psi\left[k_{1}(t, \cdot) C u_{0}\right]+\Psi\left[\left(1 * k_{1}\right)(t, \cdot) B u_{0}\right]+k_{0}(t) \Psi\left[B u_{0}\right] \\
& =N_{2}(v, H(k))(t)
\end{aligned}
$$

for $t \in[0, T]$ and $x \in(0, \ell)$ where we have set

$$
1 * k_{1}(t, x)=\int_{0}^{x} k_{1}(t, \xi) d \xi
$$

First we consider the integral equation

$$
k_{1}(t, x) \Phi\left[C u_{0}\right](x)+\Phi\left[B u_{0}\right](x) 1 * k_{1}(t, x)=f(t, x)
$$

$f$ being a prescribed function in $L^{1}((0, T) \times(0, \ell))$. Since $u_{0}$ satisfies $(2.18)$ and 1 * $k_{1}(t, 0)=0$ for any $t \in[0, T]$, integrating the first-order differential equation (3.22) for $1 * k_{1}$, we get the simple integral equation

$$
1 * k_{1}(t, x)=L f(t, x)
$$

the linear operator $L$ being defined by

$$
L f(t, x)=\int_{0}^{x} \exp \left(-\int_{\xi}^{x} \frac{\Phi\left[B u_{0}\right](r)}{\Phi\left[C u_{0}\right](r)} d r\right) \frac{f(t, \xi)}{\Phi\left[C u_{0}\right](\xi)} d \xi
$$

A direct inspection shows that function $k_{1}$ defined by

$$
k_{1}(t, x)=\frac{1}{\Phi\left[C u_{0}\right](x)} f(t, x)-\frac{\Phi\left[B u_{0}\right](x)}{\Phi\left[C u_{0}\right](x)} L f(t, x)
$$

on $[0, T] \times(0, \ell)$ satisfies (3.23) and solves the integral equation (3.22). From (3.19) and (3.25) we easily deduce that $k_{1}$ solves the equation

$$
k_{1}(t, x)=k_{0}(t) \frac{\Phi\left[B u_{0}\right](x)}{\Phi\left[C u_{0}\right](x)}\left\{L \Phi\left[B u_{0}\right](x)-1\right\}+N_{3}(v, k)(t, x)
$$


on $[0, T] \times(0, \ell)$ where

$$
\begin{aligned}
N_{3}(v, k)(t, x)= & \frac{1}{\Phi\left[C u_{0}\right](x)} \\
& \times\left\{N_{1}(v, H(k))(t, x)-\Phi\left[B u_{0}\right](x) L N_{1}(v, H(k))(t, x)\right\} .
\end{aligned}
$$

Consequently, recalling the formulas

$$
\begin{gathered}
1-L \Phi\left[B u_{0}\right](x)=\exp \left(-\int_{0}^{x} \frac{\Phi\left[B u_{0}\right](r)}{\Phi\left[C u_{0}\right](r)} d r\right) \\
1 *\left(\frac{\Phi\left[B u_{0}\right]}{\Phi\left[C u_{0}\right]}\left(L \Phi\left[B u_{0}\right]-1\right)\right)(x)=-1+\exp \left(-\int_{0}^{x} \frac{\Phi\left[B u_{0}\right](r)}{\Phi\left[C u_{0}\right](r)} d r\right)
\end{gathered}
$$

for $x \in[0, \ell]$ from (3.20) and (3.26) it is easy to derive that $k_{0}$ solves the equation (cf. $(2.18) \cdot(2.19))$ :

$$
\begin{aligned}
k_{0}(t) m_{2}\left(u_{0}\right)= & -\Psi\left[N_{3}(v, k)(t, \cdot) C u_{0}\right] \\
& -\Psi\left[1 * N_{3}(v, k)(t, \cdot) B u_{0}\right]+N_{2}(v, H(k))(t) \quad(t \in[0, T]) \\
:= & N_{4}(v, k)(t) .
\end{aligned}
$$

From property (2.19) and equations (3.26) and (3.28) we conclude that the pair $\left(k_{0}, k_{1}\right)$ solves the fixed point system

$$
\left.\begin{array}{rl}
k_{0}(t)=m_{2}\left(u_{0}\right)^{-1} N_{4}(v, k)(t) & :=N_{5}(v, k)(t) \\
k_{1}(t, x)=J_{1}\left(u_{0}\right)(x) N_{5}(v, k)(t)+N_{3}(v, k)(t, x) & :=N_{6}(v, k)(t, x)
\end{array}\right\}
$$

for $t \in[0, T]$ and $x \in(0, \ell)$ where

$$
J_{1}\left(u_{0}\right)(x)=-\frac{\Phi\left[B u_{0}\right](x)}{\Phi\left[C u_{0}\right](x)} \exp \left(-\int_{0}^{x} \frac{\Phi\left[B u_{0}\right](\xi)}{\Phi\left[C u_{0}\right](\xi)} d \xi\right)
$$

\section{Estimates for the direct wave problem}

In this section first we consider the hyperbolic direct problem

$$
\left.\begin{array}{rlrl}
D_{t}^{2} v(t, x, y)+A_{0} v(t, x, y) & =f(t, x, y) & & ((t, x, y) \in[0, T] \times \Omega) \\
v(0, x, y) & =v_{0}(x, y) & & ((x, y) \in \Omega) \\
D_{t} v(0, x, y) & =v_{1}(x, y) & & ((x, y) \in \Omega) \\
\frac{\partial v}{\partial \nu_{A_{0}}}(t, x, y) & =\frac{\partial v_{2}}{\partial \nu_{A_{0}}}(t, x, y) & & ((t, x, y) \in[0, T] \times \partial \Omega)
\end{array}\right\}
$$


where $A_{0}$ denotes the principal part of operator $A$ (cf. (1.2)). As far as the data are concerned, we make the assumptions

$$
\left.\begin{array}{l}
v_{0} \in H^{2}(\Omega), \quad v_{1} \in H^{1}(\Omega), \quad v_{2} \in \mathcal{U}^{2, \infty}(T, \Omega) \\
D_{\imath}^{2} v_{2}+A_{0} v_{2}, \quad f \in W^{1,1}\left((0, T) ; L^{2}(\Omega)\right) .
\end{array}\right\}
$$

Moreover, functions $v_{0}$ and $v_{2}$ have to satisfy the consistency conditions

$$
\frac{\partial v_{0}}{\partial \nu_{A}}(x, y)=\frac{\partial v_{2}}{\partial \nu_{A}}(0, x, y) \quad((x, y) \in \partial \Omega) .
$$

Before stating Theorem 4.1 we introduce yet the notation

$$
\|v\|_{s, \Omega}=\|v\|_{H \cdot(\Omega)} \quad(s \in \mathbb{N}) .
$$

Theorem 4.1. Under assumptions (4.2) - (4.3) the direct problem (4.1) admits a unique solution $v=L_{0}(f)+L_{1}\left(v_{0}, v_{1}, v_{2}\right) \in \mathcal{U}^{2, \infty}(T, \Omega)\left(c f\right.$. (2.15)), where $L_{0}(f)$ and $L_{1}\left(v_{0}, v_{1}, v_{2}\right)$ solve problem (4.1) with $\left(v_{0}, v_{1}, v_{2}\right)=(0,0,0)$ and $f=0$, respectively. Moreover, the linear operators $L_{0}$ and $L_{1}$ satisfy the estimate

$$
\begin{aligned}
\left\|D_{t}^{2} L_{0}(f)(t, \cdot, \cdot)\right\|_{0, \Omega}+\left\|D_{t} L_{0}(f)(t, \cdot, \cdot)\right\|_{1, \Omega} \\
\quad+\left\|L_{0}(f)(t, \cdot, \cdot)\right\|_{2, \Omega}+\left\|D_{t}^{2} L_{1}\left(v_{0}, v_{1}, v_{2}\right)(t, \cdot, \cdot)\right\|_{0, \Omega} \\
\quad+\left\|D_{t} L_{1}\left(v_{0}, v_{1}, v_{2}\right)(t, \cdot, \cdot)\right\|_{1, \Omega}+\left\|L_{1}\left(v_{0}, v_{1}, v_{2}\right)(t, \cdot, \cdot)\right\|_{2, \Omega} \\
\leq c_{1}(T)\left\{\|f\|_{W^{1,1}\left((0, t) ; L^{2}(\Omega)\right)}+\|f(0, \cdot, \cdot)\|_{0, \Omega}+\left\|v_{0}\right\|_{2, \Omega}+\left\|v_{1}\right\|_{1, \Omega}\right. \\
\left.\quad+\left\|v_{2}(t, \cdot)\right\|_{\mathcal{W}^{2, \infty}(t, \Omega)}+\left\|D_{t}^{2} v_{2}+A_{0} v_{2}\right\|_{W^{1,1}\left((0, t) ; L^{2}(\Omega)\right)}\right\}
\end{aligned}
$$

for any $t \in(0, T]$. Here $c_{1}$ denotes a positive, continuous and non-decreasing function of $T$, depending also on $\ell$ and $\omega$.

Proof. Assume that $v \in \mathcal{U}^{2, \infty}(T, \Omega)$ is a solution to problem (4.1). Then the function $z=v-v_{2}$ solves problem (4.1) with $\left(f, v_{0}, v_{1}, v_{2}\right)$ replaced by $\left(\tilde{f}, \tilde{v}_{0}, \tilde{v}_{1}, 0\right)$, where

$$
\left.\begin{array}{rl}
\tilde{f} & =f-D_{t}^{2} v_{2}-A_{0} v_{2} \\
\tilde{v}_{0} & =v_{0}-v_{2}(0, \cdot), \\
\tilde{v}_{1} & =v_{1}-D_{t} v_{2}(0, \cdot) .
\end{array}\right\}
$$

We note that the quadruplet $\left(\tilde{f}, z_{0}, z_{1}, 0\right)$ fulfils properties $(4.2)-(4.3)$ with $v_{2}=0$. An application of the Faedo-Galerkin approximation method leads to the following integral inequality for the approximating sequence $\left\{z_{m}\right\}$, where the positive constant $c_{2}$ depends on $\alpha_{1}$ and $\alpha_{2}$ only (cf. (1.5)) while the two sequences $\left\{z_{0, m}\right\}$ and $\left\{z_{1, m}\right\}$ approximate $\tilde{v}_{0}$ and $\tilde{v}_{1}$ in $H^{1}(\Omega)$ and $L^{2}(\Omega)$, respectively:

$$
\begin{aligned}
& \left\|D_{t} z_{m}(t, \cdot)\right\|_{0, \Omega}^{2}+\left\|z_{m}(t, \cdot)\right\|_{1, \Omega}^{2} \\
& \leq c_{2}\left\{\left\|z_{1, m}\right\|_{0, \Omega}^{2}+\left\|z_{0, m}\right\|_{1, \Omega}^{2}\right. \\
& \left.\quad+2 \int_{0}^{t}\|\tilde{f}(s, \cdot)\|_{0, \Omega}\left(\left\|D_{\imath} z_{m}(s, \cdot)\right\|_{0, \Omega}^{2}+\left\|z_{m}(s, \cdot)\right\|_{1, \Omega}^{2}\right)^{\frac{1}{2}} d s\right\}
\end{aligned}
$$

for a.e. $t \in(0, T)$ and all $m \in \mathbb{N}$.

We now consider the following simple variant of [1: Theorem 4.9]. 
Lemma 4.1. Let $\varphi$ be a non-negative $C([0, T])$-function, and let $b$ and $k$ be nonnegative $L^{1}(0, T)$-functions satisfying

$$
\varphi(t) \leq a+\int_{0}^{t} b(s) \varphi(s) d s+\int_{0}^{t} k(s) \varphi(s)^{p} d s \quad(t \in[0, T])
$$

where $p \in(0,1)$ and $a \geq 0$ are given constants. Then for all $t \in[0, T]$

$$
\begin{aligned}
\varphi(t) \leq & \exp \left(\int_{0}^{t} b(s) d s\right) \\
& \times\left[a^{1-p}+(1-p) \int_{0}^{t} k(s) \exp \left((p-1) \int_{0}^{s} b(\sigma) d \sigma\right) d s\right]^{\frac{1}{1-p}} .
\end{aligned}
$$

From $(4.5)-(4.6)$ (with $(p, b)=\left(\frac{1}{2}, 0\right)$ ) we easily obtain the estimate

$$
\begin{aligned}
& \left\|D_{t} z_{m}(t, \cdot)\right\|_{0, \Omega}^{2}+\left\|z_{m}(t, \cdot)\right\|_{1, \Omega}^{2} \\
& \quad \leq 2 c_{2} \max \left(1, c_{2}\right)\left\{\left\|z_{1, m}\right\|_{0, \Omega}^{2}+\left\|z_{0, m}\right\|_{1, \Omega}^{2}+\left(\int_{0}^{t}\|\tilde{f}(s, \cdot)\|_{0, \Omega} d s\right)^{2}\right\}
\end{aligned}
$$

for a.e. $t \in(0, T)$. According to this estimate, [3: Theorem 3.4.1] and [17: Corollary to Theorem 1 in Chaper 5 , Appendix] we can select a subsequence in $\left\{z_{m}\right\}_{n=1}^{+\infty}$ (still denoted by $\left.\left\{z_{m}\right\}_{m=1}^{+\infty}\right)$ and a function $z \in W^{1, \infty}\left((0, T) ; L^{2}(\Omega)\right) \cap L^{\infty}\left((0, T) ; H^{1}(\Omega)\right)$ such that

$$
\left.\begin{array}{rlrl}
z_{m} & \rightarrow z & \text { weakly } & \text { in } L^{\infty}\left((0, T) ; H^{1}(\Omega)\right) \\
D_{t} z_{m} & \rightarrow D_{\imath} z & \text { weakly * in } L^{\infty}\left((0, T) ; L^{2}(\Omega)\right)
\end{array}\right\}
$$

In particular, from these convergences we deduce that $z$ satisfies a similar estimate with the same constant $2 c_{2} \max \left(1, c_{2}\right)$.

Following the same procedure as in [3: Chapter 3/Theorem 4.1] we can prove that $z$ is the unique weak solution to problem $(4.1)$ in $L^{\infty}\left((0, T) ; H^{1}(\Omega)\right) \cap W^{1, \infty}\left((0, T) ; L^{2}(\Omega)\right)$. Moreover, a regularity procedure as in [10: Theorem 3.8.2] shows that $z$ actually belongs to $\mathcal{U}^{1, \infty}(T, \Omega)$. Then, reasoning as in [10: Theorem 5.2.1] (cf. also [13: Theorem 30.4]) we conclude that $z$ belongs to $\mathcal{U}^{2, \infty}(T, \Omega)$ and satisfies

$$
\begin{aligned}
& \left\|D_{t}^{2} z(t, \cdot)\right\|_{0, \Omega}^{2}+\left\|D_{t} z(t, \cdot)\right\|_{1, \Omega}^{2}+\|z(t, \cdot)\|_{1, \Omega}^{2} \\
& \quad \leq c_{3}(T)\left\{\|\tilde{f}\|_{W^{1,1}\left((0, t) ; L^{2}(\Omega)\right)}^{2}+\|\tilde{f}(0, \cdot)\|_{0, \Omega}^{2}+\left\|\tilde{v}_{0}\right\|_{2, \Omega}^{2}+\left\|\tilde{v_{1}}\right\|_{1, \Omega}^{2}\right\}
\end{aligned}
$$

for all $t \in[0, T]$ where the positive constant $c_{3}$ is a non-decreasing and continuous function of $T$, depending also on $\ell$ and $\omega$. Consequently, $z$ turns out to be a strong solution to problem (4.1). In particular, $A_{0} z \in C\left([0, T] ; L^{2}(\Omega)\right)$ and satisfies

$$
\begin{aligned}
& \left\|A_{0} z(t, \cdot)\right\|_{0, \Omega} \\
& \quad \leq c_{4}(T)\left\{\|\tilde{f}\|_{W^{1,1}\left((0, t) ; L^{2}(\Omega)\right)}+\|\tilde{f}(0, \cdot)\|_{0, \Omega}+\left\|\tilde{v}_{0}\right\|_{2, \Omega}+\left\|\tilde{v}_{1}\right\|_{1, \Omega}\right\}
\end{aligned}
$$


for all $t \in[0, T]$. Since $\Omega=(0, \ell) \times \omega$ is convex and $A_{0} z+z \in C\left([0, T] ; L^{2}(\Omega)\right)$, from [7: Theorem 3.2.1.3] we deduce that $z$ belongs to $C\left([0, T] ; H^{2}(\Omega)\right.$ ) and satisfies the estimates

$$
\|z(t, \cdot)\|_{2, \Omega} \leq c_{5}\left\|A_{0} z(t, \cdot)+z(t, \cdot)\right\|_{0, \Omega} \leq c_{5}\left(\left\|A_{0} z(t, \cdot)\right\|_{0, \Omega}+\|z(t, \cdot)\|_{0, \Omega}\right)
$$

for all $t \in[0, T]$, the constant $c_{5}>0$ depending only on $\ell$ and $\omega$.

Finally, from the equation $v=w+v_{2}$ we conclude that $v$ belongs to $\mathcal{U}^{2, \infty}(T, \Omega)$ and solves problem (4.1). Moreover, $v$ admits the representation $v=L_{0}(f)+L_{1}\left(v_{0} ; v_{1}, v_{2}\right)$ and, owing to estimates (4.7) - (4.9), the linear operators $L_{0}$ and $L_{1}$ satisfy (4.4). We conclude by observing that $L_{0}(f)$ and $L_{1}\left(v_{0}, v_{1}, v_{2}\right)$ solve problem $(4.1)$ with $\left(v_{0}, v_{1}, v_{2}\right)$ $=(0,0,0)$ and $f=0$, respectively

We are now in a position to solve our direct integro-differential problem (3.2) - (3.5), which we rewrite in the form

$$
D_{\imath}^{2} v(t, x, y)+A_{0} v(t, x, y)=M(v, h)(t, x, y)+D_{t} f(t, x, y)
$$

for all $(t, x, y) \in[0, T] \times \Omega$ with

$$
\left.\begin{array}{rlrl}
v(0, x, y) & =u_{1}(x, y) & & ((x, y) \in \Omega) \\
D_{t} v(0, x, y) & =-A u_{0}(x, y)+f(0, x, y) & & ((x, y) \in \Omega) \\
\frac{\partial v}{\partial \nu_{A_{0}}}(t, x, y) & =D_{t} \frac{\partial u_{2}}{\partial \nu_{A_{0}}}(t, x, y) & & ((t, x, y) \in[0, T] \times \partial \Omega) .
\end{array}\right\}
$$

Operator $M$ is defined by (cf. (3.2))

$$
\begin{aligned}
M(v, h)(t, x, y)= & -A_{5} v(t, x, y)-\int_{0}^{t} h(t-s, x) B v(s, x, y) d s \\
& -\int_{0}^{t} D_{x} h(t-s, x) C v(s, x, y) d s \\
& -D_{x} h(t, x) C u_{0}(x, y)-h(t, x) B u_{0}(x, y) .
\end{aligned}
$$

where

$$
A_{5}=a_{1}(x, y) D_{x}+\sum_{j=1}^{n-1} a_{1+j}(x, y) D_{y_{j}}+a_{0}(x, y)
$$

We then introduce the complete metric spaces of admissible unknowns $v$ and kernels $h$

$$
\begin{aligned}
& \mathcal{V}(\lambda, T)=\left\{v \in \mathcal{U}_{\lambda}^{2, \infty}(T, \Omega): v \text { satisfies }(4.11)\right\} \\
& \mathcal{H}(\lambda, T)=\left\{h \in W_{\lambda}^{1,1}\left((0, T) ; H^{1}(0, \ell)\right): h(0, \cdot)=h_{0}\right\}
\end{aligned}
$$

for all $\lambda \in \mathbb{R}_{+}$. Here $\mathcal{U}_{\lambda}^{s, \infty}(T, \Omega)$ and $W_{\lambda}^{s, 1}\left((0, T) ; H^{1}(0, \ell)\right)(s \in \mathbb{N})$ denote the weighted Banach spaces obtained from $\mathcal{U}^{s, \infty}(T, \Omega)$ (cf. (2.15)) and $W^{s, 1}\left((0, T) ; H^{1}(0, \ell)\right.$ ) by introducing the weight function $t \rightarrow \mathrm{e}^{-\lambda t}$. For instance, the norm in $W_{\lambda}^{s, 1}((0, T) ; X), X$ being any Banach space, is defined by

$$
\|u\|_{W_{\lambda}^{\prime, 1}((0, T) ; X)}=\sum_{j=0}^{s}\left\|u^{(j)}\right\|_{L^{1}\left((0, T) ; X ; \mathrm{e}^{-\lambda t d t)}\right.}=\sum_{j=0}^{s}\left\|u^{(j)}\right\|_{L_{\lambda}^{1}((0, T) ; X)} .
$$


Remark 4.1. It is immediate to check that the weighted norm above is equivalent to the usual one. Moreover, we observe that, for any pair $(v, h) \in \mathcal{V}(\lambda, T) \times \mathcal{H}(\lambda, T)$, the initial value of $M(v, h)$ is independent of $(v, h)$ : in fact (cf. $(3.16))$, it is given by

$$
\begin{aligned}
M(v, h)(0, x, y) & =-A_{5} u_{1}(x, y)-D_{x} h_{0}(x) C u_{0}(x, y)-h_{0}(x) B u_{0}(x, y) \\
& :=\widetilde{M}\left(u_{0}, u_{1}, h_{0}\right)(x, y)
\end{aligned}
$$

for all $(x, y) \in \Omega$.

Lemma 4.2. Operator $M$ maps $\mathcal{V}(\lambda, T) \times \mathcal{H}(\lambda, T)$ into $W_{\lambda}^{1, \infty}\left((0, T) ; L^{2}(\Omega)\right)$ and satisfies the following estimates for any $p \in[1,+\infty]$, where $c_{6}$ and $c_{7}$ are continuous and non-decreasing functions of the norms in $L^{\infty}(\Omega)$ of the coefficients $a_{j}(j=0, \ldots, n)$ :

$$
\begin{aligned}
& \|M(v, h)\|_{W_{\lambda}^{1, p}\left((0, T) ; L^{2}(\Omega)\right)} \\
& \quad \leq c_{6}\left(1+\left\|h_{0}\right\|_{H^{1}(0, \ell)}+\left\|u_{0}\right\|_{H^{2}(\Omega)}+\|h\|_{W_{\lambda}^{1, l}\left((0, T) ; H^{1}(0, \ell)\right)}\right)\|v\|_{U_{\lambda}^{2, p}(T, \Omega)}
\end{aligned}
$$

for all $(v, h) \in \mathcal{V}(\lambda, T) \times \mathcal{H}(\lambda, T)$ and

$$
\begin{aligned}
& \left\|M\left(v_{2}, h_{2}\right)-M\left(v_{1}, h_{1}\right)\right\|_{W_{\lambda}^{1, p}\left((0, T) ; L^{2}(\Omega)\right)} \\
& \leq c_{7}\left(1+\left\|h_{0}\right\|_{H^{1}(0, \ell)}+\left\|u_{0}\right\|_{H^{2}(\Omega)}\right. \\
& \left.\quad+\sum_{i=1}^{2}\left\|h_{i}\right\|_{W_{\lambda}^{1,1}\left((0, T) ; H^{1}(0, \ell)\right)}\right)\left\|v_{2}-v_{1}\right\|_{U_{\lambda}^{2, p}(T, \Omega)} \\
& \quad+\sum_{i=1}^{2}\left\|v_{i}\right\|_{U_{\lambda}^{2, p}(T, \Omega)}\left\|h_{2}-h_{1}\right\|_{W_{\lambda}^{1,1}\left((0, T) ; H^{1}(0, \ell)\right)}
\end{aligned}
$$

for all $\left(v_{2}, h_{2}\right),\left(v_{1}, h_{1}\right) \in \mathcal{V}(\lambda, T) \times \mathcal{H}(\lambda, T)$.

Proof. First we observe that, according to assumptions (2.1) - (2.3), (2.12) - (2.14) and (2.19) function $h_{0}$ defined by (3.16) belongs to $H^{1}(0, \ell)$. Then we note that the properties stated in the lemma are implied by definition (4.12), Young's theorem on convolutions and by the formula

$$
\begin{aligned}
& D_{t} M(v, h)(t, x, y) \\
&=-A_{5} D_{t} v(t, x, y)-\int_{0}^{t} D_{t} h(t-s, x) B v(s, x, y) d s \\
&-h_{0}(x) B v(t, x, y)-\int_{0}^{t} D_{t} D_{x} h(t-s, x) C v(s, x, y) d s \\
&-D_{x} h_{0}(x) C v(t, x, y)-D_{t} D_{x} h(t, x) C u_{0}(x, y)-D_{t} h(t, x) B u_{0}(x, y)
\end{aligned}
$$

for all $t \in(0, T)$ and all $(v, h) \in \mathcal{V}(T, \lambda) \times \mathcal{H}(T, \lambda)$. For this purpose we need the following estimates which rely on the well-known continuous embeddings $L^{\infty}(0, \ell) \cdot L^{2}(0, \ell) \hookrightarrow$ $L^{2}(0, \ell)$ ( d denotes pointwise multiplication) and $H^{1}(0, \ell) \hookrightarrow L^{\infty}(0, \ell)$;

$$
\|g f\|_{L^{2}(\Omega)} \leq\|g\|_{L^{\infty}(0, \ell)}\|f\|_{L^{2}(\Omega)} \leq c_{10}(\ell)\|g\|_{H^{1}(0, \ell)}\|f\|_{L^{2}(\Omega)}
$$


for all $f \in L^{2}(\Omega)$ and all $g \in H^{1}((0, \ell))$, and

$$
\begin{aligned}
\|g f\|_{L^{2}(\Omega)} & \leq\|g\|_{L^{2}(0, \ell)}\left(\int_{\omega}\|f(\cdot, y)\|_{L^{\infty}(0, \ell)}^{2} d y\right)^{\frac{1}{2}} \\
& \leq c_{10}(\ell)\|g\|_{L^{2}(0, \ell)}\left(\int_{\omega}\|f(\cdot, y)\|_{H^{1}(0, \ell)}^{2} d y\right)^{\frac{1}{2}} \\
& \leq c_{10}(\ell)\|g\|_{L^{2}(0, \ell)}\|f\|_{L^{2}\left(\omega_{;} H^{1}(0, \ell)\right)}
\end{aligned}
$$

for all $f \in L^{2}\left(\omega ; H^{1}(0, \ell)\right)$ and all $g \in L^{2}(0, \ell)$.

Performing standard, but boring computations, which take into account that convolutions commute with functions $t \rightarrow \mathrm{e}^{-\lambda t}$, we easily derive the assertion.

We are now in a position to solve our direct integro-differential problem. For this purpose we introduce the normed space $\mathcal{G}$ related to our data by

$$
\mathcal{G}=\left\{\begin{array}{l}
\left(f, u_{0}, u_{1}, u_{2}\right) \in W^{2,1}\left((0, T) ; L^{2}(\Omega)\right) \times H^{2}(\Omega) \times H^{2}(\Omega) \times \mathcal{U}^{4,1}(T, \Omega): \\
A u_{0}-f(0, \cdot,) \in H^{1}(\Omega), D_{t}\left(D_{\imath}^{2} u_{2}+A_{0} u_{2}\right) \in W^{2,1}\left((0, T) ; L^{2}(\Omega)\right) \\
\left(f, u_{0}, u_{1}, u_{2}\right) \text { satisfies consistency conditions }(1.13)
\end{array}\right\}
$$

The norm in $\mathcal{G}$ is defined by

$$
\begin{aligned}
\left\|\left(f, u_{0}, u_{1}, u_{2}\right)\right\|_{\mathcal{G}}= & \|f\|_{W^{2,1}\left((0, T) ; L^{2}(\Omega)\right)}+\left\|u_{0}\right\|_{H^{2}(\Omega)}+\left\|u_{1}\right\|_{H^{2}(\Omega)} \\
& +\left\|u_{2}\right\|_{u^{4,1}(T, \Omega)}+\left\|A u_{0}-f(0, \cdot, \cdot)\right\|_{H^{1}(\Omega)} \\
& +\left\|D_{t}\left(D_{t}^{2} u_{2}+A_{0} u_{2}\right)\right\|_{W^{2,1}\left((0, T)_{i} L^{2}(\Omega)\right)}
\end{aligned}
$$

Remark 4.2. It is immediate to check that $\mathcal{G}$ is actually a Banach space.

Theorem 4.2. For any $h \in \mathcal{H}(\lambda, T)$ and any quadruplet $\left(f, u_{0}, u_{1}, u_{2}\right) \in \mathcal{G}$ there exist two positive, continuous and non-decreasing functions $c_{10}$ and $c_{11}$ such that for any

$$
\lambda>\max \left(c_{9}(T), c_{10}(T)\right)\left(1+\left\|\left(f, u_{0}, u_{1}, u_{2}\right)\right\|_{\mathcal{C}}+\|h\|_{W_{\lambda}^{2 \cdot 1}\left((0, T) ; H^{1}(0, \ell)\right)}\right)
$$

problem (4.10)-(4.11) admits a unique solution $v=V(h) \in \mathcal{U}_{\lambda}^{2, \infty}(T, \Omega)$. Moreover, the nonlinear operator $V$ satisfies the following estimates for any $p \in[1,+\infty]$, where $c_{11}$ and $c_{12}$ denote positive, continuous and non-decreasing functions:

$$
\begin{aligned}
&\|V(h)\|_{u_{\lambda}^{2 . p}(T, \Omega)} \\
& \leq {\left[1-c_{9}(T)\left(1+\left\|\left(f, u_{0}, u_{1}, u_{2}\right)\right\|_{\mathcal{G}}+\|h\|_{W_{\lambda}^{1,1}\left((0, T) ; H^{1}(0, \mathcal{l})\right.}\right) \lambda^{-1}\right]^{-1} } \\
& \times \lambda^{-\frac{1}{p}} c_{11}(T)\left(1+\left\|\left(f, u_{0}, u_{1}, u_{2}\right)\right\|_{\mathcal{C}}\right)\left\|\left(f, u_{0}, u_{1}, u_{2}\right)\right\|_{\mathcal{C}}
\end{aligned}
$$


for all $h \in \mathcal{H}(\lambda, T)$ and

$$
\begin{aligned}
\| V\left(h_{2}\right) & -V\left(h_{1}\right) \|_{u_{\lambda}^{2, p}(T, \Omega)} \\
\leq & \lambda^{-\frac{1}{p}} c_{12}(T)\left[1-\max \left(c_{9}(T), c_{10}(T)\right) \lambda^{-1}\right. \\
& \left.\times\left(1+\left\|\left(f, u_{0}, u_{1}, u_{2}\right)\right\|_{\mathcal{C}}+\max _{1 \leq i \leq 2}\left\|h_{i}\right\|_{W_{\lambda}^{1,1}\left((0, T) ; H^{1}(0, \ell)\right)}\right)\right]^{-1} \\
& \times\left(1+\left\|\left(f, u_{0}, u_{1}, u_{2}\right)\right\|_{\mathcal{C}}\right)\left\|\left(f, u_{0}, u_{1}, u_{2}\right)\right\|_{\mathcal{G}}\left\|h_{2}-h_{1}\right\|_{W_{\lambda}^{1,1}\left((0, T) ; H^{1}(0, \ell)\right)}
\end{aligned}
$$

for all $h_{1}, h_{2} \in \mathcal{H}(\lambda, T)$. The latter estimate holds if $\lambda$ satisfies (4.19), with

$$
\|h\|_{W_{\lambda}^{1,1}\left((0, T) ; H^{1}(0, \ell)\right)} \quad \text { replaced with } \max _{1 \leq i \leq 2}\left\|h_{i}\right\|_{W_{\lambda}^{1,1}\left((0, T) ; H^{1}(0, \ell)\right)}
$$

Remark 4.3. According to remark 4.1 we easily conclude that the direct problem $(4.10),(4.11)$ is uniquely solvable and its solution $v$ belongs to the (non-weighted) space $\mathcal{U}^{2, p}(T, \Omega)$ and depends continuously on the relaxation kernel $h \in W^{1,1}\left((0, T) ; H^{1}(0, \ell)\right)$. The same technique used here would allow to solve the direct problem (1.1), (1.6) - (1.8) in the same space $\mathcal{U}^{2, p}(T, \Omega)$ under the simpler assumption $h \in L^{1}\left((0, T) ; H^{1}(0, \ell)\right)$, since in this case no preliminary differentiation with respect to $t$ is needed.

Proof. First we note that the quadruplet

$$
\left(M(v, h)+D_{t} f, u_{1},-A u_{0}+f(0, \cdot, \cdot), D_{t} u_{2}\right)
$$

relative to problem (4.10) - (4.11) satisfies consistency condition (4.3) for any pair $(v, h) \in \mathcal{V}(\lambda, T) \times \mathcal{H}(\lambda, T)$ by virtue of conditions (1.13) and formula (4.14). Hence, the integro-differential problem (4.10) - (4.11) is equivalent to the fixed-point equation

$$
\begin{aligned}
v & =L_{0}(M(v, h))+\left\{L_{0}\left(D_{t} f\right)+L_{1}\left(u_{1}, f(0, \cdot)-A u_{0}, D_{t} u_{2}\right)\right\} \\
& :=\mathcal{M}(v, h)+w .
\end{aligned}
$$

We note that, according to Theorem $4.1, \mathcal{M}(v, h)+w$ belongs to $\mathcal{V}(\lambda, T)$ for any pair $(v, h) \in \mathcal{V}(\lambda, T) \times \mathcal{H}(\lambda, T)$. Moreover, from Lemma 4.2 and estimate (4.4) we easily deduce the following inequalities for any $p \in[1,+\infty]$ :

$$
\begin{aligned}
& \left\|\mathcal{M}\left(v_{2}, h_{2}\right)-\mathcal{M}\left(v_{1}, h_{1}\right)\right\|_{U_{\lambda}^{2, P}(T, \Omega)} \\
& =\left\|\mathrm{e}^{-\lambda t}\left[\sum_{j=0}^{2}\left\|D_{t}^{j} \mathcal{M}\left(v_{2}, h_{2}\right)(t, \cdot)-D_{t}^{j} \mathcal{M}\left(v_{1}, h_{1}\right)(t, \cdot)\right\|_{H^{2-j}(\Omega)}\right]\right\|_{L^{p}(0, T)} \\
& \leq c_{1}(T) \|\left[\int _ { 0 } ^ { t } \mathrm { e } ^ { - \lambda ( t - s ) } \mathrm { e } ^ { - \lambda s } \left(\left\|M\left(v_{2}, h_{2}\right)(s, \cdot)-M\left(v_{1}, h_{1}\right)(s, \cdot)\right\|_{L^{2}(\Omega)}\right.\right. \\
& \left.\left.\quad+\left\|D_{t} M\left(v_{2}, h_{2}\right)(s, \cdot)-D_{t} M\left(v_{1}, h_{1}\right)(s, \cdot)\right\|_{L^{2}(\Omega)}\right) d s\right] \|_{L^{p}(0, T)} \\
& \leq c_{1}(T) \lambda^{-1}\left\|M\left(v_{2}, h_{2}\right)-M\left(v_{1}, h_{1}\right)\right\|_{W_{\lambda}^{1, p}\left((0, T) ; L^{2}(\Omega)\right)} \\
& \leq c_{10}(T) \lambda^{-1}\left[\left(1+\left\|\left(f, u_{0}, u_{1}, u_{2}\right)\right\|_{\mathcal{C}}+\max _{1 \leq i \leq 2}\left\|h_{i}\right\|_{W_{\lambda}^{1,1}\left((0, T) ; H^{1}(0, \ell)\right)}\right)\right. \\
& \left.\quad \times\left\|v_{2}-v_{1}\right\|_{U_{\lambda}^{2, p}(T, \Omega)}+\max _{1 \leq i \leq 2}\left\|v_{i}\right\|_{U_{\lambda}^{2, p}(T, \Omega)}\left\|h_{2}-h_{1}\right\|_{W_{\lambda}^{1,1}\left((0, T) ; H^{1}(0, \ell)\right)}\right]
\end{aligned}
$$


for all $\left(v_{2}, h_{2}\right),\left(v_{1}, h_{1}\right) \in \mathcal{V}(\lambda, T) \times \mathcal{H}(\lambda, T), c_{10}$ being a positive, continuous and nondecreasing function. From estimates (4.23) with $p=+\infty$ we conclude that, for any fixed $h \in \mathcal{H}(\lambda, T)$, the operator $v \rightarrow \mathcal{M}(v, h)+w$ maps $\mathcal{V}(\lambda, T)$ into itself and is a contraction mapping for any $\lambda$ satisfying (4.19). Consequently, an application of Banach's fixedpoint theorem ensures that equation (4.22) admits a unique global in time solution $v=V\left(h, f ; u_{0}, u_{1}, u_{2}\right):=V(h)$.

In order to derive estimate (4.21) we consider the following inequalities, where we make use of identity (4.14):

$$
\begin{aligned}
& \|\mathcal{M}(v, h)\|_{u_{\lambda}^{2, p}(T, \Omega)} \\
& =\left\|\left[\mathrm{e}^{-\lambda t} \sum_{j=0}^{2}\left\|D_{\imath}^{j} \mathcal{M}(v, h)(t, \cdot)\right\|_{H^{2}-j(\Omega)}\right]\right\|_{L^{p}(0, T)} \\
& \leq c_{1}(T) \|\left[\int _ { 0 } ^ { t } \mathrm { e } ^ { - \lambda ( t - s ) } \mathrm { e } ^ { - \lambda s } \left(\|M(v, h)(s, \cdot)\|_{L^{2}(\Omega)}\right.\right. \\
& \left.\left.\quad+\left\|D_{t} M(v, h)(s, \cdot)\right\|_{L^{2}(\Omega)}\right) d s+\mathrm{e}^{-\lambda \ell}\left\|\widetilde{M}\left(u_{0}, u_{1}, h_{0}\right)\right\|_{L^{2}(\Omega)}\right] \|_{L^{p}(0, T)} \\
& \leq c_{1}(T) \lambda^{-1}\|M(v, h)\|_{W_{\lambda}^{1, p}\left((0, T) ; L^{2}(\Omega)\right)}+c_{1}(T)(p \lambda)^{-\frac{1}{p}}\left\|\widetilde{M}\left(u_{0}, u_{1}, h_{0}\right)\right\|_{L^{2}(\Omega)} \\
& \leq c_{9}(T) \lambda^{-1}\left(1+\left\|\left(f, u_{0}, u_{1}, u_{2}\right)\right\|_{\mathcal{G}}+\|h\|_{W_{\lambda}^{1,1}\left((0, T) ; H^{1}(0, \ell)\right.}\right) \| v_{U_{\lambda}^{2, \infty}(T, \Omega)} \\
& \quad+c_{13}(T) \lambda^{-\frac{1}{p}}\left(1+\left\|\left(f, u_{0}, u_{1}, u_{2}\right)\right\|_{\mathcal{C}}\right)\left\|\left(f, u_{0}, u_{1}, u_{2}\right)\right\|_{\mathcal{G}}
\end{aligned}
$$

for all $(v, h) \in \mathcal{V}(\lambda, T) \times \mathcal{H}(\lambda, T), c_{13}$ being a positive, continuous and non-decreasing function. From (4.19), (4.22), (4.24) and the equation

$$
V(h)=\mathcal{M}(V(h), h)+w
$$

we easily derive estimate (4.20). In fact, for any $p \in[1,+\infty]$ we have

$$
\begin{aligned}
\left(\sum_{j=0}^{2}\left\|D_{t}^{j} w\right\|_{L_{\lambda}^{p}\left((0, T) ; H^{2-j}(\Omega)\right)}^{p}\right)^{\frac{1}{p}} & \leq(p \lambda)^{-\frac{1}{p}}\|w\|_{\mathcal{U}_{0}^{2, p}(T, \Omega)} \\
& \leq c_{1}(T)(p \lambda)^{-\frac{1}{p}}\left\|\left(f, u_{0}, u_{1}, u_{2}\right)\right\|_{\mathcal{C}}
\end{aligned}
$$

for all $\left(f, u_{0}, u_{1}, u_{2}\right) \in \mathcal{G}$. Finally, we observe that estimate (4.21) follows from the equation

$$
V\left(h_{2}\right)-V\left(h_{1}\right)=\mathcal{M}\left(V\left(h_{2}\right), h_{2}\right)-\mathcal{M}\left(V\left(h_{1}\right), h_{1}\right)
$$

and the relationships (4.20), (4.23) and (4.24) 


\section{Solving the identification problem (3.2) - (3.7)}

In this section first we introduce the complete metric space

$$
\mathcal{K}(\lambda, r, T)=\left\{\begin{array}{l}
k=\left(k_{0}, k_{1}\right) \in W_{\lambda}^{1,1}(0, T) \times W_{\lambda}^{1,1}\left((0, T) ; L^{2}(0, \ell)\right): \\
\left\|k_{0}\right\|_{W_{\lambda}^{1,1}(0, T)}+\left\|k_{1}\right\|_{W_{\lambda}^{1,1}\left((0, T) ; L^{2}(0, \ell)\right)} \leq r
\end{array}\right\}
$$

for $\lambda \in \mathbb{R}_{+}$and $r \in \mathbb{R}_{+} \cup\{+\infty\}$ and the non-linear.operator

$$
W(k)=W\left(k, f, u_{0}, u_{1}, u_{2}\right):=V\left(H(k), f, u_{0}, u_{1}, u_{2}\right) .
$$

We recall that $W(k)$ solves the operator equation (cf. (4.25) and (3.17))

$$
W(k)=\mathcal{M}(W(k), H(k))+w
$$

Assume now that $k=\left(k_{0}, k_{1}\right) \in W_{\lambda}^{1,1}(0, T) \times W_{\lambda}^{1,1}\left((0, T) ; L^{2}(0, \ell)\right)$ is a solution to problem (3.29). In particular, $k$ belongs to $\mathcal{K}(\lambda, r, T)$ for any fixed positive $r$ and any large enough $\lambda$. It is then an easy task to check that such a $k$ solves the following fixed-point problem, equivalent to (3.29),

$$
\begin{aligned}
k & \in \mathcal{K}(\lambda, r, T) \\
k_{0}(t) & =N_{5}(W(k), k)(t)=N_{5}(\mathcal{M}(W(k), H(k))+w, k)(t) \\
& :=\tilde{N}_{5}(k)(t) \\
k_{1}(t, x) & =N_{6}(W(k), k)(t, x)=N_{6}(\mathcal{M}(W(k), H(k))+w, k)(t, x) \\
& :=\tilde{N}_{6}(k)(t, x)
\end{aligned}
$$

for all $t \in[0, T]$ and $x \in \times(0, \ell)$. Moreover, we introduce the nonlinear operators (cf. $(3.6)-(3.7)$ and $(3.27)-(3.28))$

$$
\tilde{N}_{j}(k)= \begin{cases}N_{j}(\mathcal{M}(W(k), H(k))+w, H(k)) & \text { for } j=1,2 \\ N_{j}(\mathcal{M}(W(k), H(k))+w, k) & \text { for } j=3,4,5,6\end{cases}
$$

We begin by estimating operator $H$ defined in (3.17).

Lemma 5.1. Operator $H$ maps continuously $W_{\lambda}^{1,1}(0, T) \times W_{\lambda}^{1,1}\left((0, T) ; L^{2}(0, \ell)\right)$ into $W_{\lambda}^{1,1}\left((0, T) ; H^{1}(0, \ell)\right)$ and satisfies the estimate

$$
\begin{aligned}
& \left\|H\left(k_{0}, k_{1}\right)\right\|_{W_{\lambda}^{1,1}\left((0, T) ; H^{1}(0, \ell)\right)} \\
& \quad \leq \ell^{\frac{1}{2}}\left\|k_{0}\right\|_{W_{\lambda}^{1,1}(0, T)}+\left(1+\ell^{3}\right)^{\frac{1}{2}}\left\|k_{1}\right\|_{W_{\lambda}^{1,1}\left((0, T) ; L^{2}(0, \ell)\right)}
\end{aligned}
$$

Proof. It is an immediate consequence of definition (3.17) 
We introduce now the bilinear operators $\mathcal{B}$ and $\mathcal{C}$ defined on

$$
\mathcal{U}_{\lambda}^{2, \infty}(T, \Omega) \times W_{\lambda}^{1,1}\left((0, T) ; H^{1}(0, \ell)\right)
$$

(cf. (4.13)) by

$$
\begin{aligned}
& \mathcal{B}(v, h)(t, x, y)=\int_{0}^{t} h(t-s, x) B v(s, x, y) d s \\
& \mathcal{C}(v, h)(t, x, y)=\int_{0}^{t} D_{x} h(t-s, x) C v(s, x, y) d s .
\end{aligned}
$$

Lemma 5.2. The integral operators $\mathcal{B}$ and $\mathcal{C}$ are continuous from

$$
L_{\lambda}^{1}\left((0, T) ; H^{2}(\Omega)\right) \times W_{\lambda}^{1,1}\left((0, T) ; H^{1}(0, \ell)\right) \rightarrow W_{\lambda}^{1,1}\left((0, T) ; L^{2}(\Omega)\right) .
$$

Moreover, the estimates

$$
\left.\begin{array}{l}
\left\|D_{t}^{j} \mathcal{B}(v, h)\right\|_{L_{\lambda}^{1}\left((0, T) ; L^{2}(\Omega)\right)} \\
\quad \leq c_{14}\left\{j\|h(0, \cdot)\|_{L^{\infty}(0, \ell)}+\|h\|_{W_{\lambda}^{j, 1}\left((0, T) ; H^{1}(0, \ell)\right)}\right\}\|v\|_{L_{\lambda}^{1}\left((0, T) ; H^{2}(\Omega)\right)} \\
\left\|D_{t}^{j} \mathcal{C}(v, h)\right\|_{L_{\lambda}^{1}\left((0, T) ; L^{2}(\Omega)\right)} \\
\quad \leq c_{15}\left\{j\left\|D_{x} h(0, \cdot)\right\|_{L^{2}(0, \ell)}+\|h\|_{W_{\lambda}^{1,1}\left((0, T) ; H^{1}(0, \ell)\right)}\right\}\|v\|_{L_{\lambda}^{1}\left((0, T) ; H^{2}(\Omega)\right)}
\end{array}\right\}
$$

hold true for $j=0,1$ where $c_{14}$ and $c_{15}$ depend only on the norms in $L^{\infty}(\Omega)$ of the coefficients $b_{i, j}, b_{j}, b_{0}$ and $c_{j}, c_{0}$, respectively.

Proof. From the formulas

$$
D_{t}^{j} \mathcal{B}(v, h)(t, x, y)=j h(0, x) B v(t, x, y)+\int_{0}^{t} D_{\imath}^{j} h(t-s, x) B v(s, x, y) d s
$$

where $j=0,1$ we deduce the estimates

$$
\begin{aligned}
&\left\|D_{t}^{j} \mathcal{B}(v, h)(t, \cdot, \cdot)\right\|_{L^{2}(\Omega)} \\
& \leq\|B\|_{\mathcal{L}\left(H^{2}(\Omega) ; L^{2}(\Omega)\right)}\left\{j\|h(0, \cdot)\|_{L^{\infty}(0, \ell)}\|v(t, \cdot, \cdot)\|_{H^{2}(\Omega)}\right. \\
&\left.+\int_{0}^{t}\left\|D_{t}^{j} h(t-s, \cdot)\right\|_{L^{\infty}(0, \ell)}\|v(s, \cdot, \cdot)\|_{H^{2}(\Omega)} d s\right\}
\end{aligned}
$$

for all $t \in[0, T]$ which immediately imply $(5.4)_{1}$. Likewise, from

$$
D_{\imath}^{j} \mathcal{C}(v, h)(t, x, y)=j D_{x} h(0, x) C v(t, x, y)+\int_{0}^{t} D_{i}^{j} D_{x} h(t-s, x) C v(s, x, y) d s
$$

where $j=0,1$ and from (4.16) and (4.17) we deduce

$$
\begin{aligned}
\left\|D_{t}^{j} \mathcal{C}(v, h)(t, \cdot, \cdot)\right\|_{L^{2}(\Omega)} \\
\quad \leq j\left\|D_{x} h(0, \cdot)\right\|_{L^{2}((0, \ell))}\|C v(t, \cdot, \cdot)\|_{H^{1}(\Omega)} \\
\quad+\int_{0}^{\ell}\left\|D_{t}^{j} D_{x} h(t-s, \cdot)\right\|_{L^{2}((0, \ell))}\|C v(s, \cdot, \cdot)\|_{H^{1}(\Omega)} d s
\end{aligned}
$$

for all $t \in[0, T]$ which easily imply $(5.4)_{2}$ 
From now on we shall assume that our data belong the following metric space $\mathcal{D}(\rho)\left(\rho \in \mathbb{R}_{+}\right)$related to the space $\mathcal{G}$ defined by (4.18):

$$
\mathcal{D}(\rho)=\left\{\begin{array}{l}
\left(f, u_{0}, u_{1}, u_{2}, \varphi, \psi\right) \in \mathcal{G} \times \mathcal{U}^{4,1}(T,(0, \ell)) \times W^{4,1}(0, T): \\
\left(f, u_{0}, u_{1}, u_{2}, \varphi, \psi\right) \text { satisfies }(1.13)-(1.15) \text { and }(2.17) \\
\left\|\left(f, u_{0}, u_{1}, u_{2}\right)\right\|_{\mathcal{G}}+\|\varphi\|_{\mathcal{U}^{4,1}(T,(0, \ell))}+\|\psi\|_{W^{4,1}((0, T))} \leq \rho
\end{array}\right\} .
$$

From the definition (3.16) of $h_{0}$ and property (2.18) we immediately derive the estimate

$$
\left\|h_{0}\right\|_{H^{1}(0, \ell)} \leq \bar{m}_{1}^{-1} c_{16}(\rho)
$$

for all $\left(f, u_{0}, u_{1}, u_{2}, \varphi, \psi\right) \in \mathcal{D}(\rho), c_{16}$ being a continuous and non-decreasing function. Consequently, from (4.26) with $p=1$ we derive

$$
\sum_{j=0}^{2}\left\|D_{t}^{j} w\right\|_{L_{\lambda}^{1}\left((0, T) ; H^{2-j}(\Omega)\right)} \leq c_{1}(T) \rho \lambda^{-1}
$$

for all $\left(f, u_{0}, u_{1}, u_{2}, \varphi, \psi\right) \in \mathcal{D}(\rho)$.

Lemma 5.3. Let $\left(f, u_{0}, u_{1}, u_{2}, \varphi, \psi\right) \in \mathcal{D}(\rho)$ and let

$$
\lambda>\max \left(c_{9}(T), c_{10}(T)\right)\left[1+\rho+c_{17}(\ell) r\right]
$$

where $c_{17}(\ell)=\max \left(\ell^{\frac{1}{2}},\left(1+\ell^{3}\right)^{\frac{1}{2}}\right)$. Then operators $\tilde{N}_{j} \quad(j=1,2)$ defined by $(5.3)_{1}$ satisfy the following estimates for any $k, k^{1}, k^{2} \in \mathcal{K}(\lambda, r, T)$ and $j=1,2$ :

$$
\left.\begin{array}{rl}
\left\|\tilde{N}_{j}(k)-\tilde{\varphi}_{j}\right\|_{\mathcal{K}(\lambda, \infty, T)} & \leq \lambda^{-1} c_{18}\left(\lambda^{-1}, r, T, \rho\right) \\
\left.\tilde{N}^{2}\right)-\tilde{N}_{j}\left(k^{1}\right) \|_{\mathcal{K}(\lambda, \infty, T)} & \leq \lambda^{-1} c_{19}\left(\lambda^{-1}, r, T, \rho\right)\left\|k^{2}-k^{1}\right\|_{\mathcal{K}(\lambda, r, T)}
\end{array}\right\}
$$

where

$$
\left.\begin{array}{rl}
\tilde{\varphi}_{1}(t, x)= & -D_{\imath}^{3} \varphi(t, x)-D_{t} A_{1} \varphi(t, x)-\Phi_{1}\left[D_{t} \frac{\partial u_{2}}{\partial \nu_{A}}(t, \cdot)\right](x) \\
& -\Phi_{2}[w(t, \cdot)](x)+\Phi\left[D_{t} f(t, \cdot)\right](x) \\
\tilde{\varphi}_{2}(t)= & -D_{t}^{3} \psi(t)-\Psi_{1}\left[D_{t} \frac{\partial u_{2}}{\partial \nu_{A}}(t, \cdot, \cdot)\right] \\
& -\Psi_{2}[w(t, \cdot, \cdot)]+\Psi\left[D_{t} f(t, \cdot, \cdot)\right]
\end{array}\right\}
$$

for all $t \in[0, T]$ and $x \in(0, \ell)$. The functions $c_{18}$ and $c_{19}$ are non-negative, continuous and non-decreasing in each of their arguments.

Proof. First we notice that from estimates (4.20), (4.21), (4.23), (4.24) and definition (5.1) we easily derive the following estimates for any $\lambda$ satisfying (5.6) and any $\left(f, u_{0}, u_{1}, u_{2}, \varphi, \psi\right) \in \mathcal{D}(\rho)$ :

$$
\begin{aligned}
\|\mathcal{M}(W(k), H(k))\|_{U_{\lambda}^{2,1}(T, \Omega)} \\
\leq c_{9}(T)\left(1+\rho+\|H(k)\|_{W_{\lambda}^{1,1}\left((0, T) ; H^{1}(0, \ell)\right.}\right) \lambda^{-1}\|W(k)\|_{U_{\lambda}^{2,1}(T, \Omega)} \\
\quad+c_{14}(T) \rho(1+\rho) \\
\leq \lambda^{-1} \rho(1+\rho)\left\{c_{11}(T) c_{9}(T)\left(1+\rho+c_{17}(\ell) r\right)\right. \\
\left.\quad \times\left[1-c_{9}(T)\left(1+\rho+c_{17}(\ell) r\right) \lambda^{-1}\right]^{-1}+c_{14}(T)\right\}
\end{aligned}
$$


and

$$
\begin{aligned}
\left\|\mathcal{M}\left(W\left(k^{2}\right), H\left(k^{2}\right)\right)-\mathcal{M}\left(W\left(k^{1}\right), H\left(k^{1}\right)\right)\right\|_{\dot{U}_{\lambda}^{2,1}(T, \Omega)} \\
\leq c_{10}(T) \lambda^{-1}\left[\left(1+\rho+\max _{1 \leq i \leq 2}\left\|H\left(k_{i}\right)\right\|_{W_{\lambda}^{1,1}\left((0, T) ; H^{1}(0, \ell)\right.}\right)\right. \\
\quad \times\left\|W\left(k^{2}\right)-W\left(k^{1}\right)\right\|_{U_{\lambda}^{2,1}(T, \Omega)} \\
\left.\quad+\max _{1 \leq i \leq 2}\left\|W\left(k^{i}\right)\right\|_{u_{\lambda}^{2,1}(T, \Omega)}\left\|H\left(k^{2}\right)-H\left(k^{1}\right)\right\|_{W_{\lambda}^{1,1}\left((0, T) ; H^{1}(0, \ell)\right)}\right] \\
\leq c_{10}(T) \lambda^{-1}\left\|k^{2}-k^{1}\right\|_{\mathcal{X}(\lambda, r, T)}\left\{c_{12}(T)\left(1+\rho+c_{17}(\ell) r\right) c_{17}(\ell)(1+\rho) \rho\right. \\
\quad \times\left[1-\max \left(c_{9}(T), c_{10}(T)\right) \lambda^{-1}\left(1+\rho+c_{17}(\ell) r\right)\right]^{-2} \\
\left.\quad+c_{11}(T)\left[1-c_{9}(T)\left(1+\rho+c_{17}(\ell) r\right) \lambda^{-1}\right]^{-1}(1+\rho) \rho\right\}
\end{aligned}
$$

for all $k, k^{1}, k^{2} \in \mathcal{K}(\lambda, r, T)$. Consider now the following equations (cf. (5.3) $)_{1}$ ), where functions $\tilde{\varphi}_{1}$ and $\tilde{\varphi}_{2}$ are defined by (5.8):

$$
\begin{aligned}
\tilde{N}_{1}(k)(t, x)= & \widetilde{\varphi}_{1}(t, x)-\Phi_{2}[\mathcal{M}(W(k), H(k))(t, \cdot)](x) \\
& -\Phi[\mathcal{B}(\mathcal{M}(W(k), H(k)))(t, \cdot)](x) \\
& -\Phi[\mathcal{C}(\mathcal{M}(W(k), H(k)))(t, \cdot)](x) \\
& -\Phi[\mathcal{B}(w, H(k))(t, \cdot)](x)-\Phi[\mathcal{C}(w, H(k))(t, \cdot)](x) \\
= & : \tilde{\varphi}_{1}(t, x)+\tilde{N}_{7}(k)(t, x)
\end{aligned}
$$

and

$$
\begin{aligned}
\tilde{N}_{2}(k)(t)= & \widetilde{\varphi}_{2}(t)-\Psi_{2}[\mathcal{B}(\mathcal{M}(W(k), H(k))(t, \cdot, \cdot)] \\
& -\Psi[\mathcal{B}(\mathcal{M}(w, H(k))(t, \cdot, \cdot)] \\
& -\Psi[\mathcal{C}(\mathcal{M}(W(k), H(k)))(t, \cdot, \cdot)] \\
& -\Psi[\mathcal{B}(w, H(k))(t, \cdot, \cdot)]-\Psi[\mathcal{C}(w, H(k))(t, \cdot, \cdot)] \\
= & : \tilde{\varphi}_{2}(t, x)+\widetilde{N}_{8}(k)(t, x)
\end{aligned}
$$

for all $t \in[0, T]$ and all $x \in(0, \ell)$, as well as the identity

$$
\begin{aligned}
\Pi\left[\mathcal{E}\left(\mathcal{M}\left(W\left(k^{2}\right), H\left(k^{2}\right)\right), H\left(k^{2}\right)\right)\right]-\Pi\left[\mathcal{E}\left(\mathcal{M}\left(W\left(k^{1}\right), H\left(k^{1}\right)\right), H\left(k^{1}\right)\right)\right] \\
=\Pi\left[\mathcal{E}\left(\mathcal{M}\left(W\left(k^{2}\right), H\left(k^{2}\right)\right)-\mathcal{M}\left(W\left(k^{1}\right), H\left(k^{1}\right)\right), H\left(k^{2}\right)\right)\right] \\
\quad+\Pi\left[\mathcal{E}\left(\mathcal{M}\left(W\left(k^{1}\right), H\left(k^{1}\right)\right), H\left(k^{2}\right)-H\left(k^{1}\right)\right)\right]
\end{aligned}
$$

for all $\Pi \in\left\{\Phi, \Phi_{2}, \Psi, \Psi_{2}\right\}$ and $\mathcal{E} \in\{\mathcal{B}, \mathcal{C}, \mathcal{I}\}, \mathcal{I}$ denoting the identity operator. Finally, from (5.9) - (5.13), (5.5), Lemma 5.2 and assumptions (2.4) and (2.8) - (2.10) we easily derive estimates (5.7) 
Lemma 5.4. For any $T \in \mathbb{R}_{+}$the operator $L$ defined by (3.24) belongs to

$$
\mathcal{L}\left(W_{\lambda}^{1,1}\left((0, T) ; L^{2}(0, \ell)\right) ; W_{\lambda}^{1,1}\left((0, T) ; H^{1}(0, \ell)\right)\right)
$$

and its norm does not exceed $c_{20}\left(\rho, \bar{m}_{1}^{-1}\right), c_{20}$ being a continuous and non-decreasing function in each of its arguments.

Proof. It immediately follows from definition (3.24) mates

Lemma 5.5. The operators $\tilde{N}_{j}(j=3,4,5,6)$ defined by $(5.3)_{2}$ satisfy the esti-

$$
\left.\begin{array}{c}
\left\|\tilde{N}_{j}(k)-\tilde{\varphi}_{j}\right\|_{\mathcal{K}(\lambda, \infty, T)} \leq \lambda^{-1} c_{20}\left(\lambda^{-1}, r, T, \rho\right) \\
\left\|\tilde{N}_{j}\left(k^{2}\right)-\tilde{N}_{j}\left(k^{1}\right)\right\|_{\mathcal{K}(\lambda, \infty, T)} \leq \lambda^{-1} c_{21}\left(\lambda^{-1}, r, T, \rho\right)\left\|k^{2}-k^{1}\right\|_{\mathcal{K}(\lambda, r, T)}
\end{array}\right\}
$$

for all.k, $k^{1}, k^{2} \in \mathcal{K}(\lambda, r, T)$ with

$$
\begin{aligned}
\tilde{\varphi}_{3}(t, x) & =\frac{1}{\Phi\left[C u_{0}\right](x)}\left\{\tilde{\varphi}_{1}(t, x)-\Phi\left[B u_{0}\right](x) L \tilde{\varphi}_{1}(t, x)\right\} \\
\widetilde{\varphi}_{4}(t) & =-\Psi\left[\tilde{\varphi}_{3}(t, \cdot) C u_{0}\right]-\Psi\left[1 * \widetilde{\varphi}_{3}(t, \cdot) B u_{0}\right]+\widetilde{\varphi}_{2}(t) \\
\widetilde{\varphi}_{5}(t) & =m_{2}\left(u_{0}\right)^{-1} \widetilde{\varphi}_{4}(t) \\
\tilde{\varphi}_{6}(t, x) & =J_{1}\left(u_{0}\right)(x) \tilde{\varphi}_{5}(t)+\widetilde{\varphi}_{3}(t, x) .
\end{aligned}
$$

The functions $c_{20}$ and $c_{21}$ are non-negative, continuous and non-decreasing in each of their arguments.

Proof. From definitions $(5.3)_{2},(3.27)$ - (3.29) we easily deduce the following relationships, where $t \in[0, T]$ and $x \in(0, \ell)$ (cf. (5.11) - (5.12) and (5.15)):

$$
\begin{aligned}
\tilde{N}_{3}(v, k)(t, x) & =\widetilde{\varphi}_{3}(t, x)+\frac{1}{\Phi\left[C u_{0}\right](x)}\left\{\tilde{N}_{7}(k)(t, x)-\Phi\left[B u_{0}\right](x) L \tilde{N}_{7}(k)(t, x)\right\} \\
& =: \widetilde{\varphi}_{3}(t, x)+\tilde{N}_{9}(k)(t, x) \\
\tilde{N}_{4}(k)(t) & =\widetilde{\varphi}_{4}(t)-\Psi\left[\tilde{N}_{9}(k)(t, \cdot) C u_{0}\right]-\Psi\left[1 * \tilde{N}_{9}(k)(t, \cdot) B u_{0}\right]+\widetilde{N}_{8}(k)(t) \\
& =: \tilde{\varphi}_{4}(t)+\tilde{N}_{10}(k)(t) \\
\tilde{N}_{5}(k)(t) & =\widetilde{\varphi}_{4}(t)+m_{2}\left(u_{0}\right)^{-1} \tilde{N}_{10}(k)(t):=\tilde{\varphi}_{5}(t)+\tilde{N}_{11}(k)(t) \\
\tilde{N}_{6}(k)(t, x) & =J_{1}\left(u_{0}\right)(x) \tilde{N}_{11}(k)(t)+\tilde{N}_{9}(k)(t, x) .
\end{aligned}
$$

Finally, from these relationships, equations $\tilde{N}_{j}(k)-\tilde{\varphi}_{j}=\tilde{N}_{6+j}(k) \quad(j=1,2)$ and Lemmas 5.1 - 5.4 we easily derive estimates $(5.14)$

We are now in a position to prove Theorem 2.1 . 
Proof of Theorem 2.1. From definitions (5.8) and (5.15) and estimates (4.16) and (4.17) we easily deduce that functions $\widetilde{\varphi}_{1}, \widetilde{\varphi}_{3}, \tilde{\varphi}_{6}$ and $\widetilde{\varphi}_{2}, \tilde{\varphi}_{4}, \widetilde{\varphi}_{5}$ belong to $W_{\lambda}^{1,1}((0, T)$; $\left.L^{2}(0, \ell)\right)$ and $W_{\lambda}^{1,1}(0, T)$, respectively. We now observe that Lemma 5.5 easily implies

$$
\begin{aligned}
& \left\|\tilde{N}_{5}(k)\right\|_{W_{\lambda}^{1,1}(0, T)}+\left\|\tilde{N}_{6}(k)\right\|_{W_{\lambda}^{1,1}\left((0, T) ; L^{2}(0, \ell)\right)} \\
& \quad \leq\left\|\tilde{\varphi}_{5}\right\|_{W_{\lambda}^{1,1}(0, T)}+\left\|\widetilde{\varphi}_{6}\right\|_{W_{\lambda}^{1,1}\left((0, T) ; L^{2}(0, \ell)\right)}+2 \lambda^{-1} c_{20}\left(\lambda^{-1}, r, T, \rho\right) \\
& \leq\left\|\tilde{\varphi}_{5}\right\|_{W^{1,1}(0, T)}+\left\|\tilde{\varphi}_{6}\right\|_{W^{1,1}\left((0, T) ; L^{2}(0, \ell)\right)}+2 \lambda^{-1} c_{20}\left(\lambda^{-1}, r, T, \rho\right)
\end{aligned}
$$

for all $k \in \mathcal{X}(\lambda, r, T)$ and

$$
\begin{gathered}
\left\|\tilde{N}_{5}\left(k^{2}\right)-\tilde{N}_{5}\left(k^{1}\right)\right\|_{W_{\lambda}^{1,1}(0, T)}+\left\|\tilde{N}_{6}\left(k^{2}\right)-\tilde{N}_{6}\left(k^{1}\right)\right\|_{W_{\lambda}^{1,1}\left((0, T) ; L^{2}(0, \ell)\right)} \\
\leq 2 \lambda^{-1} c_{21}\left(\lambda^{-1}, r, T, \rho\right)\left\|k^{2}-k^{1}\right\|_{\mathcal{K}(\lambda, r, T)}
\end{gathered}
$$

for all $k^{1}, k^{2} \in \mathcal{K}(\lambda, r, T)$. Then for any fixed

$$
r>r_{0}:=\left\|\tilde{\varphi}_{5}\right\|_{W^{1,1}(0, T)}+\left\|\tilde{\varphi}_{6}\right\|_{W^{1,1}\left((0, T) ; L^{2}(0, \ell)\right)}
$$

choose $\lambda \in \mathbb{R}_{+}$to be a solution to the system of inequalities

$$
\left.\begin{array}{r}
\left\|\widetilde{\varphi}_{5}\right\|_{W^{1,1}((0, T))}+\left\|\tilde{\varphi}_{6}\right\|_{W^{1,1}\left((0, T) ; L^{2}(0, \ell)\right)}+\lambda^{-1} c_{20}\left(\lambda^{-1}, r, T, \rho\right) \leq r \\
2 \lambda^{-1} c_{21}\left(\lambda^{-1}, r, T, \rho\right)<1
\end{array}\right\} .
$$

Then the vector operator $\tilde{N}=\left(\tilde{N}_{5}, \tilde{N}_{6}\right)$ maps $\mathcal{K}(\lambda, r, T)$ into itself and turns out to be a contraction mapping in $\mathcal{K}(\lambda, r, T)$, for any fixed $r>r_{0}$ and $\lambda$ large enough. Consequently, problem (5.2) admits a unique solution in $W_{\lambda}^{1,1}(0, T) \times W_{\lambda}^{1,1}\left((0, T) ; L^{2}(0\right.$, $\ell)$ )

Outline of the proof of Theorem 2.2. We limit ourselves to stating that the proof is a consequence of the techniques developed in Sections 4 and 5 if we take into account the following fixed-point system (cf. (5.2))

$$
\left.\begin{array}{rl}
k, \tilde{k} & \in \mathcal{K}(\lambda, r, T) \\
k_{0}(t)-\widetilde{k}_{0}(t) & =\tilde{N}_{5}(k)(t)-\tilde{N}_{5}(\widetilde{k})(t) \quad(t \in[0, T]) \\
k_{1}(t, x)-\widetilde{k}_{1}(t, x) & =\tilde{N}_{6}(k)(t, x)-\tilde{N}_{6}(\tilde{k})(t, x) \quad((t, x) \in[0, T] \times(0, \ell))
\end{array}\right\}
$$

Consequently the basic task consists in estimating the right hand sides in $-(5.16)$ in terms of the difference $k-\tilde{k}$ in the weighted Banach space $\mathcal{K}(\lambda, r, T)$ 


\section{Analysing the specific additional conditions (1.11), (1.12)}

In the case of the specific operators $\Phi$ and $\Psi$ (cf. (1.11) - (1.12)) the results in Theorem 2.1 hold true, if we assume that the functions $\lambda$ and $\rho$ satisfy the properties

$$
\lambda, \rho \in C^{0,1}(\bar{\Omega}) \quad \text { and } \quad D_{x}^{2} \lambda \in C(\bar{\Omega}) .
$$

Remark 6.1. We recall that (cf. remark 2.1) the further consistency conditions (2.17) may occur when the vector valued function $\left(a_{1,2}, \ldots, a_{1, n}\right)$ vanishes everywhere on $\{0, \ell\} \times \omega$ and the operators $\Phi_{0, k}(k=0,1)$ reduce to the null operator. The latter property occurs when $D_{x} \lambda=0$ on $\{0, \ell\} \times \omega$, since

$$
\Phi_{0, k}[v]=\int_{\omega} D_{x} \lambda(k \ell, y) v(k \ell, y) d y \quad(k=0,1)
$$

We observe now that, in the present case, conditions (2.18) and (2.19) can be rewritten in the explicit forms

$$
\left|\int_{\omega} \lambda(x, y) C u_{0}(x, y) d y\right| \geq \bar{m}_{1} \quad(x \in[0, \ell])
$$

and

$$
\begin{aligned}
\mid \int_{(0, \ell) \times \omega} \rho(x, y)( & \left.B u_{0}(x, y)-\frac{\int_{\omega} \lambda(x, y) B u_{0}(x, \eta) d \eta}{\int_{\omega} \lambda(x, y) C u_{0}(x, \eta) d \eta} C u_{0}(x, y)\right) \\
& \times \exp \left(-\int_{0}^{x} \frac{\int_{\omega} \lambda(x, \eta) B u_{0}(\xi, \eta) d \eta}{\int_{\omega} \lambda(x, \eta) C u_{0}(\xi, \eta) d \eta} d \xi\right) d x d y \mid \geq \bar{m}_{2}
\end{aligned}
$$

for some constants $\bar{m}_{1}>0$ and $\bar{m}_{2}>0$.

Lemma 6.1. Conditions (2.4)-(2.10) are satisfied under assumptions (2.1)-(2.3), (6.1) and the following

$$
\sum_{j=1}^{n-1} \nu_{j}(y) a_{1,1+j}(x, y)=0
$$

on $(0, l) \times \partial \omega, \nu$ denoting the outward normal unit vector related to $\partial \omega$.

Remark 6.2. Property (6.2) is trivially satisfied if we assume that the coefficients $a_{1, j}(j=2, \ldots, n)$ vanish on $(0, l) \times \partial \omega$. Consequently, Theorems 2.1 and 2.2 holds true with conditions (2.4)-(2.10) being replaced with (6.1), (6.2). sition

Proof of Lemma 6.1. First we observe that the operator $A$ admits the decompo-

$$
A=\sum_{j=1}^{5} A_{j}
$$


where

$$
\left.\begin{array}{l}
A_{1}=-D_{x}\left[a_{1,1}(x) D_{x}\right] \\
A_{2}=-\sum_{j=1}^{n-1} D_{x}\left[a_{1,1+j}(x, y) D_{y_{j}}\right] \\
A_{3}=-\sum_{i=1}^{n-1} D_{y_{i}}\left[a_{1+i, 1}(x, y) D_{x}\right] \\
A_{4}=-\sum_{i, j=1}^{n-1} D_{y_{i}}\left[a_{1+i, 1+j}(x, y) D_{y_{j}}\right] \\
A_{5}=a_{1}(x, y) D_{x}+\sum_{j=1}^{n-1} a_{1+j}(x, y) D_{y_{j}}+a_{0}(x, y)
\end{array}\right\}
$$

Differentiating under the integral sign, integrating by parts and using the identity $g D_{x} f=D_{x}(f g)-f D_{x} g$ from definition (1.11) and assumptions (2.12), (6.1) and (6.2) we easily get the equations for any $w \in H^{2}(\Omega), \nu$ standing for the outward normal unit vector related to $\partial \omega$ :

$$
\begin{aligned}
\Phi\left[A_{1} w\right](x)= & A_{1} \Phi[w](x)+2 a_{1,1}(x) \int_{\omega} D_{x} \lambda(x, y) D_{x} w(x, y) d y \\
& +\int_{\omega}\left[a_{1,1}(x) D_{x}^{2} \lambda(x, y)+a_{1,1}^{\prime}(x) D_{x} \lambda(x, y)\right] w(x, y) d y \\
\Phi\left[A_{2} w\right](x)= & -\sum_{j=1}^{n-1} \int_{\omega} \lambda(x, y) a_{1,1+j}(x, y) D_{y_{j}} D_{x} w(x, y) d y \\
& -\sum_{j=1}^{n-1} \int_{\omega} \lambda(x, y) D_{x} a_{1,1+j}(x, y) D_{y_{j}} w(x, y) d y \\
= & \sum_{j=1}^{n-1} \int_{\omega}\left[D_{y_{j}}\left(a_{1,1+j} \lambda\right)(x, y) D_{x} w(x, y)\right. \\
& \left.-\lambda(x, y) D_{x} a_{1,1+j}(x, y) D_{y_{j}} w(x, y)\right] d y \\
& \sum_{i=1}^{n-1} \int_{\omega} a_{1+i, 1}(x, y) D_{y_{i}} \lambda(x, y) D_{x} w(x, y) d y \\
& +\sum_{i, j=1}^{n-1} \int_{\omega} a_{1+i, 1+j}(x, y) D_{y_{i}} \lambda(x, y) D_{y_{j}} w(x, y) d y \\
\Phi\left[A_{4} w\right](x)= & -\int_{\partial \omega} \lambda(x, y) \sum_{i, j=1}^{n-1} \nu_{i}(y) a_{1+i, 1+j}(x, y) D_{y j} w(x, y) d \sigma(y) \\
&
\end{aligned}
$$

From (6.4) we easily derive that $\Phi$ admits decomposition (2.4), $\Phi_{1}$ and $\Phi_{2}$ being defined, 
for $z \in L^{2}(\partial \Omega)$ and $w \in H^{1}(\Omega)$, respectively, by

$$
\Phi_{1}[z](x)=-\sum_{j=1}^{n-1} \int_{\partial \omega} \lambda(x, y) z(x, y) d \sigma(y)
$$

and

$$
\begin{aligned}
\Phi_{2}[w] & (x) \\
= & \int_{w}\left\{2 a_{1,1}(x) D_{x} \lambda(x, y) D_{x} w(x, y)\right. \\
& +\left[a_{1,1}(x) D_{x}^{2} \lambda(x, y)+a_{1,1}^{\prime}(x) D_{x} \lambda(x, y)\right] w(x, y) \\
& +\sum_{j=1}^{n-1}\left[D_{y_{j}}\left(a_{1,1+j} \lambda\right)(x, y) D_{x} w(x, y)-\lambda(x, y) D_{x} a_{1,1+j}(x, y) D_{y j} w(x, y)\right] \\
& +\sum_{i=1}^{n-1} a_{1+i, 1}(x, y) D_{y_{i}} \lambda(x, y) D_{x} w(x, y) \\
& +\sum_{i, j=1}^{n-1} a_{1+i, 1+j}(x, y) D_{y_{i}} \lambda(x, y) D_{y j} w(x, y) \\
& \quad+\lambda(x, y) a_{1}(x, y) D_{x} w(x, y) \\
& \left.\quad+\lambda(x, y) \sum_{j=1}^{n-1} a_{1+j}(x, y) D_{y_{j}} w(x, y)+\lambda(x, y) a_{0}(x, y) w(x, y)\right\} d y .
\end{aligned}
$$

From here we can easily check that $\Phi_{1}$ and $\Phi_{2}$ enjoy the properties in (2.4) - (2.9).

We now observe that the functional $\Psi$ defined by (1.12) fulfil the properties in (2.4) and (2.10) by virtue of (6.1). More exactly, for any $z \in L^{2}(\partial \Omega)$ and $w \in H^{1}(\Omega)$, respectively, we get

$$
\Psi_{1}[z]=\sum_{k=0}^{1}(-1)^{k} \int_{\omega} \rho(k \ell, y) z(k \ell, y) d y-\sum_{i=1}^{n-1} \int_{(0, \ell) \times \partial \omega} \rho(x, y) z(x, y) d x d \sigma(y)
$$

and

$$
\begin{aligned}
\Psi_{2}[w]= & \int_{\Omega} D_{x} \rho(x, y)\left[a_{1,1}(x) D_{x} w(x, y)+\sum_{j=1}^{n-1} a_{1,1+j}(x, y) D_{y_{j}} w(x, y)\right] d x d y \\
& +\int_{\Omega}\left\{\sum _ { i = 1 } ^ { n - 1 } D _ { y _ { i } } \rho ( x , y ) \left[a_{1+i, 1}(x, y) D_{x} w(x, y)\right.\right. \\
& \left.+\sum_{j=1}^{n-1} a_{i+i, 1+j}(x, y) D_{y_{j}} w(x, y)\right] \\
& +\rho(x, y)\left[a_{1}(x, y) D_{x} w(x, y)\right. \\
& \left.\left.+\sum_{j=1}^{n-1} a_{1+j}(x, y) D_{y_{j}} w(x, y)+a_{0}(x, y) w(x, y)\right]\right\} d x d y
\end{aligned}
$$


Thus the statement is proved

\section{References}

[1] Bainov, D. and P. Simeonov: Integral Inequalities and Applications (Mathematics and its Applications: vol. 57). Dordrecht: Kluwer Acad. Publ. 1992.

[2] Cavaterra, C. and A. Lorenzi: An identification problem for the Maxwell equations in a non homogeneous dispersive medium. Diff. Int. Eq. 8 (1995), $1167-1190$.

[3] Duvaut, G. and J. L. Lions: Inequalities in Mechanics and Physics. Berlin - Heidelberg - New York: Springer-Verlag 1976.

[4] Grasselli, M.: On an inverse problem for a linear hyperbolic integro-differential equation. Forum Math. 6 (1994), 83 - 110.

[5] Grasselli, M., Kabanikhin, S. I. and A. Lorenzi: An inverse integro-differential problem arising in Geophysics Part I. Sib. Math. J. 33 (1992), 415 - 426.

[6] Grasselli, M., Kabanikhin, S. I. and A. Lorenzi: An inverse integro-differential problem arising in Geophysics. Part II. Nonlin. Anal.: Theory, Methods and Appl. 15 (1990), 283 $-298$.

[7] Grisvard, P.: Elliptic Problems in Non-Smooth Domains (Monographs and Studies in Mathematics: Vol. 24). Boston: Pitman 1985.

[8] Janno, J.: Inverse problems for determining monotone weakly singular relaxation kernels in viscoelasticity. Preprint.

[9] Janno, J. and von Woldersdorf, L.: Inverse problems for identification of memory kernels in viscoelasticity. Math. Meth. Appl. Sci. 20 (1997), $291-314$.

[10] Lions, J. L. and E. Magenes: Non-Homogeneous Boundary Value Problems and Applications. Volumes I and II. Berlin - Heidelberg - New York: Springer Verlag 1972.

[11] Lorenzi, A.: Direct and inverse integro-differential Maxwell problems for dispersive media related to cylindrical domains. In: Inverse Problems in Diffusion Processes, Proceedings of the 1994 GAMM-SIAM Symposium (eds.: H. Engl \& W. Rundell). SIAM 1995, pp. 42. -89 .

[12] Lorenzi, A. and V. G. Yakhno: An identification problem related to an isotropic nonhomogeneous stratified viscolelastic cylindrical body. J. Inv. Ill-Posed Prob. 5 (1997), 29 53.

[13] Wloka, J.: Partial Differential Equations. Cambridge at al.: Univ. Press 1987.

[14] von Woldersdorf, L.: On identification of memory kernels in linear viscoelasticity. Math. Nachr. 161 (1993), $203-217$.

[15] von Woldersdorf, L.: Identification of memory kernels and Riemann-Hilbert problems. Part I. Preprint. Freiberg: TU Bergakad., Preprint 95-10.

[16] von Woldersdorf, L.: Identification of memory kernels and Riemann-Hilbert problems. Part II. Preprint. Freiberg: TU Bergakad., Preprint 96-09.

[17] Yosida, K.: Functional Analysis. Berlin - Heidelberg - New York: Springer-Verlag 1969. 